\title{
THE PUZZLE OF PARTIAL MIGRATION: ADAPTIVE DYNAMICS AND EVOLUTIONARY GAME THEORY PERSPECTIVES
}

\author{
PATRICK DE LEENHEER, ANUSHAYA MOHAPATRA, HALEY A. OHMS, DAVID A. LYTLE, AND J.M. CUSHING
}

\begin{abstract}
We consider the phenomenon of partial migration which is exhibited by populations in which some individuals migrate between habitats during their lifetime, but others do not. First, using an adaptive dynamics approach, we show that partial migration can be explained on the basis of negative density dependence in the per capita fertilities alone, provided that this density dependence is attenuated for increasing abundances of the subtypes that make up the population. We present an exact formula for the optimal proportion of migrants which is expressed in terms of the vital rates of migrant and non-migrant subtypes only. We show that this allocation strategy is both an evolutionary stable strategy (ESS) as well as a convergence stable strategy (CSS). To establish the former, we generalize the classical notion of an ESS because it is based on invasion exponents obtained from linearization arguments, which fail to capture the stabilizing effects of the nonlinear density dependence. These results clarify precisely when the notion of a "weak ESS", as proposed in [24] for a related model, is a genuine ESS. Secondly, we use an evolutionary game theory approach, and confirm, once again, that partial migration can be attributed to negative density dependence alone. In this context, the result holds even when density dependence is not attenuated. In this case, the optimal allocation strategy towards migrants is the same as the ESS stemming from the analysis based on the adaptive dynamics.

The key feature of the population models considered here is that they are monotone dynamical systems, which enables a a rather comprehensive mathematical analysis.
\end{abstract}

\section{Contents}

1. Introduction 2

2. A population model for migrants and non-migrants with fixed allocation strategy 3

2.1. Population model 3

2.2. Density dependence assumptions 3

2.3. The Basic Reproduction number $\quad 4$

2.4. Global Stability for the population model 4

3. Adaptive dynamics: generalizing the definition of ESS and CSS 5

3.1. The coupled resident-mutant model $\quad 5$

3.2. Linear invasion analysis $\quad 7$

3.3. Nonlinear invasion analysis: $\phi^{*}$ is an ESS and a CSS for convex fertility functions 9

3.4. Parametrizing models for salmonid species 11

4. Evolutionary dynamics $r$

5. Comparing adaptive dynamics and evolutionary game theoretic approaches 13

6. Conclusions 14

7. Acknowledgments 14

8. Appendix 215

8.1. Proof of Theorem $2.1 \quad 15$

8.2. Dynamics along a dominant eigenvector 17

8.3. At most one positive fixed point 19

8.4. Proof of Theorem 4.2 20

$\begin{array}{ll}\text { References } & 22\end{array}$

Date: October 16, 2016.

2000 Mathematics Subject Classification. 37.

Key words and phrases. Partial Migration, Density Dependence, Basic reproduction number, Monotone systems, Global stability, Adaptive Dynamics, Evolutionary Stable Strategy, Evolutionary Game Theory.

1

(C) 2016. This manuscript version is made available under the Elsevier user license

http://www.elsevier.com/open-access/userlicense/1.0/ 


\section{INTRODUCTION}

Partial migration is the phenomenon in which some individuals of a population migrate between habitats, whereas others remain in a single habitat during their entire lifetime [5]. There are many examples of populations that exhibit partial migration. In many bird species for instance, a fraction of the population remains on site year-round, whereas the remaining fractions migrates towards warmer or more Southern latitudes to overwinter [28]. Certain fish populations also display partial migration. For example, in salmonids species a fraction of individuals migrate to the ocean before returning to spawn, and others spend their entire life in the stream $[17,9]$.

There has been a lot of interest into why and how nature has developed such a complex system in which populations consist of a mix of migratory and non-migratory individuals. Several mechanisms have been implicated, including genetic control, density-dependence, and exogenous stochastic effects in environmental variablessee $[23,16,18,5,29,40,24]$ and references therein.

In this paper, motivated primarily by partial migration in the salmonid Oncorhynchus mykiss, we take an adaptive dynamics approach, and show that negative density dependence in the per capita fertilities alone can explain this intriguing phenomenon, provided that the negative density-dependence is attenuated with increasing subtype abundances. Our results confirm and extend those of [18, 24], although the analysis in these papers is based on different population models. Moreover, we clarify the notion of a "weak Evolutionary Stable Strategy", which was proposed in [24], and uncover a condition on the nature of the density dependence that guarantees that it is in fact a true Evolutionary Stable Strategy (or ESS). But to achieve this, we must first revisit and extend the definition of an ESS as found in most references $[10,6,34,8]$. This is because the classical definition is based on a linear stability analysis near a fixed point (or, more generally near an invariant set) of a particular nonlinear dynamical system that models the interaction between the candidate ESS and a mutant strategist. As it turns out, here such a linear stability analysis near the fixed point corresponding to a candidate ESS, is inconclusive because the linearization is critical. However, it is possible to perform a nonlinear stability analysis of this fixed point, avoiding center manifold based approaches, but instead exploiting an important feature of the dynamical system, known as $K$-monotonicity [15].

Furthermore, we show that this ESS is also a Convergent Stable Strategy (CSS). This implies that this strategy is an evolutionary attractor for the adaptive dynamics. An ESS which is also a CSS is one of the strongest notions in adaptive dynamics; not only can such a strategy resist invasion by mutants strategists (by being an ESS), but the strategy value adopted by the population will also eventually converge to this strategy in the evolutionary process, via consecutive fitness-increasing mutant substitutions.

Another aspect of our work is that we derive a formula for the ESS in terms of the demographic model parameters for the migrant and non-migrant populations. This formula can be used to predict the ESS value, whenever the basic demographic parameters of the migrant and non-migrant populations are known. Additionally, this formula may be used to determine some of these demographic parameters in case they are not known, provided that a reliable estimate of the ESS value exists, possibly based on field or lab data.

To assess if our findings depend on the specific principles upon which the theory of adaptive dynamics is built, we also investigate whether partial migration can be explained using ideas from evolutionary game theory [44]. We find that the exact same ESS found using adaptive dynamics, is also the optimal allocation strategy towards migrants in this case. This provides solid theoretical evidence for the specific ESS value predicted by both modeling frameworks. An interesting difference between the two approaches, is that density dependence of the fertilities does no longer have to be attenuated in the evolutionary game setup. Whether or not attenuation in density dependence can be relaxed in the realm of adaptive dynamics, is currently unknown. 


\section{A POPULATION MODEL FOR MIGRANTS AND NON-MIGRANTS WITH FIXED ALLOCATION STRATEGY}

2.1. Population model. We consider the following density-dependent model.

$$
\left(\begin{array}{c}
x_{1}(t+1) \\
x_{M}(t+1) \\
x_{N}(t+1)
\end{array}\right)=\left(\begin{array}{ccc}
0 & f_{M}\left(x_{M}(t)\right) & f_{N}\left(x_{N}(t)\right) \\
\phi s_{M} & 0 & 0 \\
(1-\phi) s_{N} & 0 & 0
\end{array}\right)\left(\begin{array}{c}
x_{1}(t) \\
x_{M}(t) \\
x_{N}(t)
\end{array}\right)
$$

where $x_{1}(t), x_{M}(t)$ and $x_{N}(t)$ are non-negative real numbers, respectively representing the abundances of eggs, migrant adults and non-migrant adults at time $t$, where $t$ is a non-negative integer. A fraction $\phi \in[0,1]$ of eggs at time $t$ will become migrant adults, provided they survive a season, which is captured by the survival probability $s_{M} \in(0,1]$ in the model. Similarly, a fraction $1-\phi$ of eggs will become nonmigrant adults, after surviving a season, with survival probability $s_{N} \in(0,1]$. The parameter $\phi$ represents an allocation strategy whereby each morph (migrant or non-migrant) produces offspring that can become either type of morphs. In this way, the population is completely integrated and does not represent two separately evolving populations. For now, we assume that $\phi$ is fixed, although later on, when taking an adaptive dynamics perspective, we will think of $\phi$ as a varying strategy value that will be subject to evolution.

2.2. Density dependence assumptions. We assume that the number of eggs for both migrant and non-migrant adults is negatively affected by their respective abundances. This implicitly assumes that the migrant abundance does not affect the fertility of non-migrants, and vice versa. This is based on the biological system of steelhead (migrants) and rainbow trout (non-migrants) (both of the species O. mykiss), because the two morphs have segregated spawning times or habitats [27].

Mathematically, we make the following assumptions:

(H1) $f_{M}:[0, \infty) \rightarrow(0, \infty)$ and $f_{N}:[0, \infty) \rightarrow(0,+\infty)$ are smooth functions, with negative derivatives, i.e. $f_{M}^{\prime}(z)<0$, and $f_{N}^{\prime}(z)<0$, for all $z \geq 0$. We set $f_{M, \infty}=\lim _{z \rightarrow \infty} f_{M}(z)$ and $f_{N, \infty}=\lim _{z \rightarrow \infty} f_{N}(z)$, for appropriate non-negative constants $f_{M, \infty}$ and $f_{N, \infty}$.

(H2) $g_{M}(z):=f_{M}(z) z$ and $g_{N}(z):=f_{N}(z) z$ have positive derivatives, i.e. $g_{M}^{\prime}(z)>0$, and $g_{N}^{\prime}(z)>$ 0 , for all $z \geq 0$.

(H2) expresses that, although the per capita fertilities decrease as the adult abundances increase (by (H1)), the total fertilities of migrants and residents are in fact increasing.

An important consequence of condition (H2), is that it ensures that system (1) is monotone. This means that if $x(0) \leq y(0)$ (the latter inequality for the two vectors means that all 3 corresponding components of these vectors are ordered accordingly), then $x(1) \leq y(1)$, for any given pair of non-negative initial population vectors $x(0)$ and $y(0)$. To prove this, we apply the fundamental theorem of calculus, in conjunction with the fact that the Jacobian matrix in any state of system (1) is a non-negative matrix by condition (H2):

$$
y(1)-x(1)=T(y(0))-T(x(0))=\int_{0}^{1} J(t y(0)+(1-t) x(0))(y(0)-x(0)) d t \geq 0,
$$

where $T$ denotes the map on the right-hand side of (1), and $J$ is the Jacobian matrix of system (1) (i.e., the derivative of the map $T$ ).

Examples: Several well-known fertility functions satisfy the two hypotheses $(\mathbf{H 1})-(\mathbf{H} 2)$ :

- The Beverton-Holt function [2]

$$
f_{B}(z)=\frac{a_{B}}{1+b_{B} z}
$$

where $a_{B}$ and $b_{B}$ are arbitrary positive constants, serves as our main example of a fertility function that satisfies all the above conditions. Indeed, $f_{B}(z)$ is a smoothly decreasing function of $z \geq 0$ with zero limit as $z \rightarrow \infty$, hence it satisfies $(\mathbf{H 1})$. The function $g_{B}(z)=z f_{B}(z)$ has derivative

$$
g_{B}^{\prime}(z)=\frac{a_{B}}{\left(1+b_{B} z\right)^{2}},
$$


which is positive for $z \geq 0$, hence it satisfies $(\mathbf{H 2})$.

- The Hassell function [14] generalizes the Beverton-Holt function:

$$
f_{H}(z)=\frac{a_{H}}{\left(1+b_{H} z\right)^{c_{H}}},
$$

where $a_{H}$ and $b_{H}$ are still arbitrary positive constants, but an additional positive parameter $c_{H}$ is introduced (for $c_{H}=1$, we recover the Beverton-Holt function). The function $f_{H}(z)$ is a smoothly decreasing function of $z \geq 0$ with zero limit as $z \rightarrow \infty$, hence it satisfies $(\mathbf{H 1})$. The function $g_{H}(z)=z f_{H}(z)$ has derivative

$$
g_{H}^{\prime}(z)=\frac{a_{H}\left(1+\left(1-c_{H}\right) b_{H} z\right)}{\left(1+b_{H} z\right)^{c_{H}+1}},
$$

which is positive for $z \geq 0$, provided that

$$
0<c_{H} \leq 1
$$

whence satisfies (H2).

On the other hand, the Ricker function [32]:

$$
f_{R}(z)=\mathrm{e}^{r(1-z / k)}
$$

where $r$ and $k$ are positive parameters satisfies (H1), but does not satisfy (H2). Indeed, the function $g_{R}(z)=z f_{R}(z)$ has derivative

$$
g_{R}^{\prime}(z)=\mathrm{e}^{r(1-z / k)}\left(1-\frac{r}{k} z\right)
$$

which changes sign when $z$ crosses through the value $k / r$. Therefore, the function $g_{R}(z)$ is not monotonically increasing for $z \geq 0$, hence fails to satisfy $(\mathbf{H 2})$.

2.3. The Basic Reproduction number. Model (1) can be re-written more compactly in vector form as

$$
x(t+1)=A_{1}(x(t), \phi) x(t),
$$

where

$$
x=\left(\begin{array}{c}
x_{1} \\
x_{M} \\
x_{N}
\end{array}\right), \text { and } A_{1}(x, \phi)=\left(\begin{array}{ccc}
0 & f_{M}\left(x_{M}\right) & f_{N}\left(x_{N}\right) \\
\phi s_{M} & 0 & 0 \\
(1-\phi) s_{N} & 0 & 0
\end{array}\right) .
$$

By splitting $A_{1}(x, \phi)$ as:

$$
A_{1}(x, \phi)=F+T \text {, where } F=\left(\begin{array}{ccc}
0 & f_{M}\left(x_{M}\right) & f_{N}\left(x_{N}\right) \\
0 & 0 & 0 \\
0 & 0 & 0
\end{array}\right) \text {, and } T=\left(\begin{array}{ccc}
0 & 0 & 0 \\
\phi s_{M} & 0 & 0 \\
(1-\phi) s_{N} & 0 & 0
\end{array}\right),
$$

we can associate the basic reproduction number to the non-negative matrix $A_{1}(x, \phi)$ in the usual way $[7,4,22,1]$ :

$$
R_{0}(x, \phi):=\rho\left(F(I-T)^{-1}\right)=\phi s_{M} f_{M}\left(x_{M}\right)+(1-\phi) s_{N} f_{N}\left(x_{N}\right), \text { for every }(x, \phi) \in \mathbb{R}_{+}^{3} \times[0,1] .
$$

Here, $\rho\left(F(I-T)^{-1}\right)$ denotes the spectral radius of $F(I-T)^{-1}$.

For convenience, we also define

$$
R_{0}(\infty, \phi)=\phi s_{M} f_{M, \infty}+(1-\phi) s_{N} f_{N, \infty}, \text { for every } \phi \in[0,1] .
$$

2.4. Global Stability for the population model. The main result, which we prove in the Appendix, is that populations with a fixed allocation strategy $\phi$ will settle at a globally stable fixed point, provided that the basic reproduction number near the extinction fixed point is larger than 1 :

Theorem 2.1. Assume that $(\mathbf{H 1})-(\mathbf{H 2})$ holds for system (1), and that the basic reproduction numbers satisfy:

$$
R_{0}(\infty, \phi)<1<R_{0}(0, \phi), \text { for all } \phi \in[0,1] \text {. }
$$

Then the following holds: 
(1) For all $\phi \in(0,1)$, system (1) has a unique, positive fixed point $x^{*}(\phi)$ which is linearly stable, and which attracts all positive solutions of (1).

(2) If $\phi=0$, then system (1) has a unique, non-zero fixed point $\left(\tilde{x}_{1}, 0, \tilde{x}_{N}\right)$, where $\tilde{x}_{N}>0$ is the unique positive solution to the equation $s_{N} f_{N}(z)=1$, and $\tilde{x}_{1}=\tilde{x}_{N} / s_{N}$. This fixed point is linearly stable, and attracts all positive solutions of system (1).

(3) If $\phi=1$, then system (1) has a unique, non-zero fixed point $\left(\hat{x}_{1}, \hat{x}_{M}, 0\right)$, where $\hat{x}_{M}>0$ is the unique positive solution to the equation $s_{M} f_{M}(z)=1$, and $\hat{x}_{1}=\hat{x}_{M} / s_{M}$. This fixed point is linearly stable, and attracts all positive solutions of system (1).

The function $\phi \rightarrow x^{*}(\phi)$ is smooth, and $\lim _{\phi \rightarrow 0} x^{*}(\phi)=\left(\tilde{x}_{1}, 0, \tilde{x}_{N}\right)$ and $\lim _{\phi \rightarrow 1} x^{*}(\phi)=\left(\hat{x}_{1}, \hat{x}_{M}, 0\right)$.

Remark 2.2. Notice that condition (4) is equivalent to:

$$
s_{M} f_{M}(0)>1, s_{N} f_{N}(0)>1 \text {, and } s_{M} f_{M, \infty}<1, s_{N} f_{N, \infty}<1 .
$$

because $R_{0}(0, \phi)\left(R_{0}(\infty, \phi)\right)$ is a convex combination of the numbers $s_{M} f_{M}(0)$ and $s_{N} f_{N}(0)\left(s_{M} f_{M, \infty}\right.$ and $\left.s_{N} f_{N, \infty}\right)$.

Also note that $f_{M, \infty}=f_{R, \infty}=0$ in case $f_{M}$ and $f_{R}$ are Beverton-Holt or Hassell functions, and hence the condition that $R_{0}(\infty, \phi)<1$ is automatically satisfied, because $R_{0}(\infty, \phi)=0$.

\section{AdAPtive DyNAMiCs: GENERALIZING THE DEFINITION OF ESS AND CSS}

Theorem 2.1 shows that for a given allocation strategy $\phi$, there will be a unique globally stable fixed point. This result does not, however, explain which value of $\phi$ is adopted in natural populations. One possible choice would be to pick the value of $\phi$ that maximizes the basic reproduction number $R_{0}(0, \phi)$. Formula (3) shows that the latter is a linear function of the variable $\phi$, which would suggest that $\phi$ must take on one of its extreme values, namely zero or one, at least if the extremal values $s_{M} f_{M}(0)$ and $s_{N} f_{N}(0)$ of the function $R_{0}(0, \phi)$ are different (if they are the same, then $R_{0}(0, \phi)$ is a constant function, hence every $\phi$ in $[0,1]$ would be a maxmizer). This would imply that either only migrants (if $\phi=1$ is the maximizer), or only non-migrants (if $\phi=0$ is the maximizer) would occur, and this would obviously contradict that a mix of migrants and non-migrants is present in natural populations.

Instead of the above argument, here we shall take an adaptive dynamics approach to address this question. We will show that there exists a unique evolutionary stable strategy (ESS) $\phi^{*}$, corresponding to a non-extreme value of the strategy parameter $\phi$, i.e. $\phi^{*}$ will belong to $(0,1)$. Moreover, we will show that $\phi^{*}$ is also a converging stable strategy (CSS), making $\phi^{*}$ an evolutionary stable attractor for the adaptive dynamics. The latter property provides theoretical evidence for how the ESS $\phi^{*}$ has evolved dynamically. Moreover, we shall derive an analytical formula for the ESS $\phi^{*}$ in terms of the model's demographic parameters. This is relevant to natural populations for which these parameters are known (e.g. from field work or from lab experiments), because the formula can be used to predict the ESS $\phi^{*}$.

3.1. The coupled resident-mutant model. We consider a resident population that uses strategy value $\phi$, and assume invasion by a mutant population using strategy value $\phi^{\prime} \neq \phi$. The resulting dynamical population model takes the following form:

$$
X(t+1)=A(X(t)) X(t),
$$

where

$$
\begin{gathered}
X(t)=\left(\begin{array}{c}
\text { resident egg abundance }\left(x_{1}(t)\right) \\
\text { resident migrant adult abundance }\left(x_{M}(t)\right) \\
\text { resident non-migrant adult abundance }\left(x_{N}(t)\right) \\
\text { mutant egg abundance }\left(y_{1}(t)\right) \\
\text { mutant migrant adult abundance }\left(y_{M}(t)\right) \\
\text { mutant non-migrant adult abundance }\left(y_{N}(t)\right)
\end{array}\right), \\
A(X(t))=\left(\begin{array}{cc}
A_{1}(x(t)+y(t), \phi) & 0 \\
0 & A_{1}\left(x(t)+y(t), \phi^{\prime}\right)
\end{array}\right)
\end{gathered}
$$


with

$$
x=\left(\begin{array}{c}
x_{1} \\
x_{M} \\
x_{N}
\end{array}\right), y=\left(\begin{array}{c}
y_{1} \\
y_{M} \\
y_{N}
\end{array}\right), \text { and } A_{1}(x, \phi)=\left(\begin{array}{ccc}
0 & f_{M}\left(x_{M}\right) & f_{N}\left(x_{N}\right) \\
\phi s_{M} & 0 & 0 \\
(1-\phi) s_{N} & 0 & 0
\end{array}\right)
$$

The specific form of the interaction matrix $A(X)$ implies that the density dependence of the fertilities by migrants, only depends on the total (resident plus mutant, or $x_{M}+y_{M}$ ) adult migrant abundance, and not on the non-migrant abundance. Once again, this is motivated by the species $O$. mykiss, in which migrants and non-migrants spawn in segregated habitats, thereby avoiding competitive effects with each other in this process. The fact that density dependence is assumed to depend on the total abundances, expresses that residents and mutants of each morph (migrant and non-migrant) spawn in the same areas, hence experience the detrimental competitive effects of each other's presence. Another important feature of this model is that it assumes that there is no inter-mating between residents and mutants. Indeed, resident adults will only generate resident eggs, and mutant adults will only generate mutant eggs. This is a strong assumption favoring assortative mating, although this is questionable. However, in the context of adaptive dynamics, it is a commonly made assumption. One problem when trying to relax this assumption, is that additional biological knowledge is required, which may not be available. For example, when a resident and mutant adult mate, the nature of their offspring (resident or mutant) needs to be specified. This requires the introduction of yet another allocation strategy parameter for the probability that this offspring becomes a resident. This probability depends on complex and poorly known genetic and environmental factors. We avoid such difficulties by making an assortative mating assumption, yet are fully aware of its limitations. The model studied here can serve as a benchmark to compare the behavior of future models to, which incorporate the possibility of inter-mating.

We assume that all assumptions $(\mathbf{H 1})-(\mathbf{H} 2)$ and condition (4) of Theorem 2.1 continue to hold here for system (5). Hence, for each $\phi$ in $[0,1]$, system (5) has a fixed point $X^{*}(\phi)=\left(\begin{array}{c}x^{*}(\phi) \\ 0\end{array}\right)$, where $x^{*}(\phi)=$ $\left(\begin{array}{c}x_{1}^{*}(\phi) \\ x_{M}^{*}(\phi) \\ x_{N}^{*}(\phi)\end{array}\right)$ is the unique nonzero fixed point of system (1) featured in Theorem 2.1.

Definition 3.1. We say that $\phi^{*}$ in $[0,1]$ is an evolutionary stable strategy (ESS) if $X^{*}\left(\phi^{*}\right)$ is a locally asymptotically stable fixed point of system (5) for all $\phi^{\prime} \neq \phi^{*}$ in some neighborhood of $\phi^{*}$.

This notion captures that if the resident population has adopted an ESS, then it cannot be invaded by mutants that use nearby strategies. Contrary to its terminology, an ESS merely represents a fixed point for the adaptive dynamics. This does not necessarily mean that an ESS possesses any kind of stability properties for the adaptive dynamics. An ESS that exhibits certain stability features for the adaptive dynamics, requires the introduction of another concept:

Definition 3.2. We say that $\phi^{*}$ in $[0,1]$ is a convergence stable strategy (CSS) if there is a neighborhood $N$ of $\phi^{*}$ such that $X^{*}\left(\phi^{*}\right)$ is not an asymptotically stable fixed point of system (5) for all pairs $\left(\phi, \phi^{\prime}\right)$ with $\phi$ in $N$ that satisfy that either $\phi<\phi^{\prime}<\phi^{*}$ or $\phi^{*}<\phi^{\prime}<\phi$, but an asymptotically stable fixed point of system (5) for all pairs $\left(\phi, \phi^{\prime}\right)$ with $\phi$ in $N$ that satisfy that either $\phi^{\prime}<\phi<\phi^{*}$ or $\phi^{*}<\phi<\phi^{\prime}$.

The intuitive idea behind this definition is as follows: Suppose that the resident has adopted a strategy that is nearby, but distinct from a CSS. Suppose also that a mutant is introduced whose strategy is farther away from the CSS value. This mutant will then fail to invade the environment occupied by the resident. But over time, a mutant may arise whose strategy value is closer to the CSS value. Such a mutant will be able to successfully invade the resident's environment. Iterating this process, yields a sequence of successfully invading mutants whose strategies converge monotonically towards the CSS.

Definitions 3.1 and 3.2 are more general than the corresponding ones usually found in the literature, as they cover the latter. For instance, no reference is made to any kind of fitness function, which is what is normally done when defining ESS and CSS. The main reason for the more general definitions proposed here, is that whereas notions of ESS and CSS in the literature are invariably based on linearization arguments 
near fixed points of coupled models, we encounter a situation here where such arguments are inconclusive. In particular, we find that the linearization of the coupled model (5) near the boundary fixed point $X^{*}\left(\phi^{*}\right)$, where $\phi^{*}$ is the candidate ESS, is critical due to the occurrence of 2 eigenvalues having modulus equal to 1. According to the classical ESS definition based on linearization arguments, $\phi^{*}$ would not be called on ESS. However, a more detailed non-linear analysis reveals that the fixed point $X^{*}\left(\phi^{*}\right)$ is locally asymptotically stable for all values $\phi^{\prime} \neq \phi^{*}$. In other words, no mutant $\phi^{\prime} \neq \phi^{*}$ can successfully invade the resident $\phi^{*}$, and this is exactly the key property exhibited by an ESS as captured by Definition 3.1.

3.2. Linear invasion analysis. To investigate whether the mutant can successfully invade the resident population, we fix a (resident,mutant) strategy pair $\left(\phi, \phi^{\prime}\right)$ in $[0,1] \times[0,1]$, and linearize system $(5)$ near the fixed point $X^{*}(\phi)$. By Theorem 2.1, the upper diagonal block of the linearization near $X^{*}(\phi)$ is a stable matrix, and thus we focus on the $3 \times 3$ matrix in the lower-diagonal block, which takes the form:

$$
A_{1}\left(x^{*}(\phi), \phi^{\prime}\right)=\left(\begin{array}{ccc}
0 & f_{M}\left(x_{M}^{*}(\phi)\right) & f_{N}\left(x_{N}^{*}(\phi)\right) \\
\phi^{\prime} s_{M} & 0 & 0 \\
\left(1-\phi^{\prime}\right) s_{N} & 0 & 0
\end{array}\right)
$$

The mutant can successfully invade if the dominant eigenvalue of this matrix is larger than 1 , and it cannot invade if it has dominant eigenvalue less than 1 . Since this dominant eigenvalue and $R_{0}\left(x^{*}(\phi), \phi^{\prime}\right)$ are always on the same side of 1 as shown in [22], the success or failure of invasion by the mutant can be determined by checking the sign of:

$$
W\left(\phi, \phi^{\prime}\right):=R_{0}\left(x^{*}(\phi), \phi^{\prime}\right)-1=\phi^{\prime} s_{M} f_{M}\left(x_{M}^{*}(\phi)\right)+\left(1-\phi^{\prime}\right) s_{N} f_{N}\left(x_{N}^{*}(\phi)\right)-1,
$$

where we have used the formula (3). In the context of adaptive dynamics, the function $W\left(\phi, \phi^{\prime}\right)$ is often referred to as the fitness of the mutant adopting strategy $\phi^{\prime}$ in an environment occupied by a resident population adopting strategy $\phi$. If $W\left(\phi, \phi^{\prime}\right)>0$, then the mutant can successfully invade; if $W\left(\phi, \phi^{\prime}\right)<0$, then the mutant fails to invade.

We next simplify the function $W\left(\phi, \phi^{\prime}\right)$ by using the fact that $x^{*}(\phi)$ is the unique non-zero fixed point of system (1), or equivalently of system (2). Then the Perron-Frobenius Theorem implies in particular that the dominant eigenvalue of $A_{1}\left(x^{*}(\phi), \phi\right)$ is equal to one, and hence by [22] that:

$$
R_{0}\left(x^{*}(\phi), \phi\right)=\phi s_{M} f_{M}\left(x_{M}^{*}(\phi)\right)+(1-\phi) s_{N} f_{N}\left(x_{N}^{*}(\phi)\right)=1 .
$$

Therefore,

$$
W\left(\phi, \phi^{\prime}\right)=\left(\phi^{\prime}-\phi\right)\left(s_{M} f_{M}\left(x_{M}^{*}(\phi)\right)-s_{N} f_{N}\left(x_{N}^{*}(\phi)\right)\right) .
$$

This simplification reveals that $W\left(\phi, \phi^{\prime}\right)$ is the product of $\left(\phi^{\prime}-\phi\right)$ and a function that depends only on $\phi$. We further investigate the latter function, and focus on which value(s) of $\phi$ in $[0,1]$, this function is zero. This happens if and only if $s_{M}\left(f_{M}\left(x_{M}^{*}(\phi)\right)\right)=s_{N} f_{N}\left(x_{N}^{*}(\phi)\right)$. Since $x^{*}(\phi)$ is a non-zero fixed point of $(1)$, there holds that

$$
x_{1}^{*}(\phi)=x_{1}^{*}(\phi)\left(\phi s_{M} f_{M}\left(x_{M}^{*}(\phi)+(1-\phi) s_{N} f_{N}\left(x_{N}^{*}(\phi)\right)\right) .\right.
$$

Theorem 2.1 shows that $x_{1}^{*}(\phi)>0$ for all $\phi$ in $[0,1]$, and therefore $s_{M}\left(f_{M}\left(x_{M}^{*}(\phi)\right)\right)=s_{N} f_{N}\left(x_{N}^{*}(\phi)\right)$ holds, if and only if

$$
s_{M} f_{M}\left(x_{M}^{*}(\phi)\right)=1=s_{N} f_{N}\left(x_{N}^{*}(\phi)\right) .
$$

But this is equivalent to

$$
x_{M}^{*}(\phi)=\hat{x}_{M} \text { and } x_{N}^{*}(\phi)=\tilde{x}_{N},
$$

where we recall that $\hat{x}_{M}$ and $\tilde{x}_{N}$ are the respective unique solutions of the equations $s_{M} f_{M}(z)=1$ and $s_{N} f_{N}(z)=1$. Since $x^{*}(\phi)$ is a non-zero fixed point of system (1) with $x_{1}^{*}(\phi)>0$, this is equivalent to

$$
\frac{x_{M}^{*}(\phi)}{x_{N}^{*}(\phi)}=\frac{\hat{x}_{M}}{\tilde{x}_{N}}=\frac{s_{M}}{s_{N}} \frac{\phi}{1-\phi},
$$

The equation on the right can now be solved for $\phi$, and this yields the following unique solution in $[0,1]$ :

$$
\phi^{*}=\frac{\hat{x}_{M} / \tilde{x}_{N}}{\hat{x}_{M} / \tilde{x}_{N}+s_{M} / s_{N}} \text {. }
$$




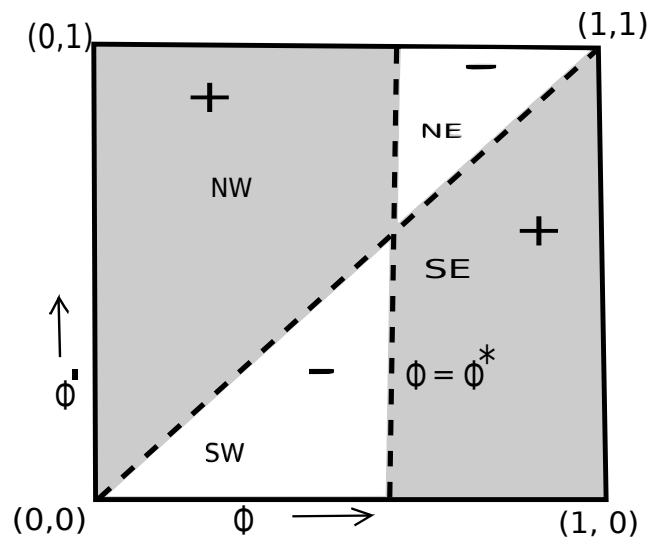

Figure 1. Pairwise invasibility plot (PIP): $W\left(\phi, \phi^{\prime}\right)$ is the fitness of a mutant strategist $\phi^{\prime}$ in an environment set by a resident strategist $\phi$, which is obtained from a linear stability analysis of the fixed point $X^{*}(\phi)$ of system (5). $W\left(\phi, \phi^{\prime}\right)=0$ if $\left(\phi, \phi^{\prime}\right)$ belongs to one of the two dashed lines; $W\left(\phi, \phi^{\prime}\right)>0$ if $\left(\phi, \phi^{\prime}\right)$ belongs to the NW or SE region (dark); $W\left(\phi, \phi^{\prime}\right)<00$ if $\left(\phi, \phi^{\prime}\right)$ belongs to the NE or SW region (light).

The formula (8) clearly shows that $\phi^{*}$ belongs to $(0,1)$, which corresponds to a non-extreme allocation strategy, exhibiting a mix of migrants and non-migrants. We have established that $W\left(\phi, \phi^{\prime}\right)=0$ if and only if

$$
\phi=\phi^{\prime} \text { or } \phi=\phi^{*} .
$$

It is now relatively straightforward to describe the sign of the function $W\left(\phi, \phi^{\prime}\right)$ for all pairs $\left(\phi, \phi^{\prime}\right)$ in $[0,1] \times[0,1]$, as depicted in Figure 1. Indeed, $W\left(\phi, \phi^{\prime}\right)$ is a continuous function, which is zero if and only if either the pair $\left(\phi, \phi^{\prime}\right)$ belongs to the diagonal where $\phi=\phi^{\prime}$, or to the vertical line through $\phi^{*}$. These two lines divide the square $[0,1] \times[0,1]$ in four open regions, where the sign of the function $W$ is either positive or negative. We label these -perhaps a bit inaccurately, compared to the familiar compass directions- by NE, SE, SW and NW, see Figure 1. The sign of $W\left(\phi, \phi^{\prime}\right)$ for pairs $\left(\phi, \phi^{\prime}\right)$ in the SE region is the same as the sign of $W(1,0)=-\left(1-s_{N} f_{N}(0)\right)$, which is positive by Remark 2.2. Similarly, $W(0,1)=s_{M} f_{M}(0)-1$ is positive as well, and thus $W\left(\phi, \phi^{\prime}\right)>0$ in the NW region. The sign of $W\left(\phi, \phi^{\prime}\right)$ for pairs $\left(\phi, \phi^{\prime}\right)$ in the NE region is the same as the sign of $W(\phi, 1)$, where $\phi$ is an arbitrary value in the open interval $\left(\phi^{*}, 1\right)$. Since $W$ can be factored as in $(7)$, the sign of $W(\phi, 1)$ is equal to the sign of the second factor $s_{M} f_{M}\left(x_{M}^{*}(\phi)\right)-s_{N} f_{N}\left(x_{N}^{*}(\phi)\right)$, which is continuous in $\phi$. The analysis above has shown that the latter function can only be zero when $\phi=\phi^{*}$, and since $\phi>\phi^{*}$, it follows that the sign of this second factor is equal to the sign of $s_{M} f_{M}\left(x_{M}^{*}(1)\right)-s_{N} f_{N}\left(x_{N}^{*}(1)\right)=1-s_{N} f_{N}(0)$, which is negative by Remark 2.2. Thus, $W\left(\phi, \phi^{\prime}\right)$ is negative for all pairs $\left(\phi, \phi^{\prime}\right)$ in the NE region. A similar argument shows that $W\left(\phi, \phi^{\prime}\right)$ is negative for all pairs $\left(\phi, \phi^{\prime}\right)$ in the SW region.

Remark 3.3. It is rather remarkable that the Pairwise Invasibility Plot looks exactly the same as the corresponding PIPs in [24] and [18], although the latter papers consider different population models. The underlying population model considered here has 3 state variables (eggs, migrant and non-migrant adults), and differs from the one in [24] where there is only a single state variable for the total (migrant plus non-migrant) number of adults. Moreover, the model in [24] assumes that density dependence takes place in the survival probabilities $s_{M}$ and $s_{N}$, not in fertilities $f_{M}$ and $f_{N}$. And one more difference, is that in addition to density dependence, there is also explicit trait dependence on the parameter $\phi$ of the survival probabilities. Despite all these differences, the PIP of both models look exactly the same. In fact, the same is true for the model considered by [18], where the underlying population model resembles more closely the one considered here. Indeed, that model has 2 state variables (for migrant and non-migrant adults), and considers only density dependence (no frequency dependence) in the fertilities, but not in the survival probabilities. On the other hand, the model deviates from the one presented here in the specific way in which density dependence occurs for the migrants, namely by assuming that migrants experience 
density dependence based on the sum of migrant and non-migrant adults. In our models, the subtypes only experience density dependence from individuals of the same subtype. But once again, the PIP obtained in [18] looks identical to the one obtained here as well. Thus, it appears that partial migration can be based on the existence of a unique ESS which is also a CSS for a wide variety of models. However, at this stage in the analysis we have not yet established that $\phi^{*}$ is indeed an ESS. This will be achieved in the following subsection, using a nonlinear local stability analysis. Such a nonlinear analysis has not been carried out in [24] or [18], which explain the proposal in [24] to refer to $\phi^{*}$ as a 'weak ESS'. Our analysis will reveal that this so-called weak ESS, is in fact a true ESS.

3.3. Nonlinear invasion analysis: $\phi^{*}$ is an ESS and a CSS for convex fertility functions. The linear invasion analysis performed above, shows that the only candidate ESS is $\phi^{*}$, given by formula (8). However, since $W\left(\phi^{*}, \phi^{\prime}\right)=0$ for all $\phi^{\prime}$ in $[0,1]$, it is not yet possible to conclude that $\phi^{*}$ is in fact an ESS in the sense of Definition 3.1. To achieve this, we will subject system (5) to a local, but nonlinear stability analysis near the fixed point $X\left(\phi^{*}\right)$ for arbitrary values of the mutant strategy $\phi^{\prime} \neq \phi^{*}$. Our main result is that $\phi^{*}$ is indeed an ESS, as well as a CSS, provided that the fertility functions $f_{M}$ and $f_{N}$ are strictly convex, which is the case when they are both Beverton-Holt or Hassell functions as we will show later.

Theorem 3.4. Assume that the conditions of Theorem 2.1 hold. Let $\phi^{*}$ be given by (8), and suppose that $\phi^{\prime} \in(0,1)$, but that $\phi^{\prime} \neq \phi^{*}$. If

$$
\text { (C) } f_{M}^{\prime \prime}\left(x_{M}^{*}\left(\phi^{*}\right)\right)>0 \text { and } f_{N}^{\prime \prime}\left(x_{N}^{*}\left(\phi^{*}\right)\right)>0 \text {, }
$$

then the fixed point $X^{*}\left(\phi^{*}\right)$ of system (5) is locally asymptotically stable, and hence $\phi^{*}$ is an ESS. Moreover, $\phi^{*}$ is the unique ESS in $[0,1]$, and it is also a CSS.

Proof. The linear invasion analysis in the subsection above clearly shows that the only candidate for an ESS is $\phi^{*}$. Indeed, if $\phi \neq \phi^{*}$, then there always exist mutant strategies $\phi^{\prime} \neq \phi$, but near $\phi$, such that $W\left(\phi, \phi^{\prime}\right)>0$. This implies that the fixed point $X^{*}(\phi)$ is a (linearly) unstable fixed point of system (5), and hence such a $\phi$ cannot be an ESS in the sense of Definition 3.1. We also note that the linear invasion analysis above shows that $\phi^{*}$ is always a CSS, by verifying that the conditions in Definition 3.2 hold, which can be seen quite easily from Figure 1 .

Thus, we are left with proving that $\phi^{*}$ is an ESS when $(\mathbf{C})$ holds. We will do this by showing that the fixed point $X^{*}\left(\phi^{*}\right)$ of system (5), is locally asymptotically stable whenever $\phi^{\prime} \in(0,1)$, yet $\phi^{\prime} \neq \phi^{*}$.

A key property of system (5) is that it is monotone [15] with respect to the partial order on $\mathbb{R}^{6}$ induced by the cone $K=\mathbb{R}_{+}^{3} \times\left(-\mathbb{R}_{+}^{3}\right)$; we will refer to this by saying that system (5) is $K$-monotone. This cone $K$ generates the partial order $\leq_{K}$ on $\mathbb{R}_{+}^{6}$, which is defined by declaring that $X \leq_{K} Y$ if and only if the vector $Y-X$ belongs to $K$. $K$-monotonicity of system (5) is then defined as follows:

$$
\text { If } X \leq_{K} Y \text {, then } A(X) X \leq_{K} A(Y) Y \text {. }
$$

Thus, $K$-monotonicity simply expresses that 2 solutions of system (5) that are ordered initially,will preserve the order. That system (5) is indeed $K$-monotone follows from the assumptions (H1) and (H2), as shown in $[15]$.

By means of the linear coordinate change

$$
\tilde{X}=X-X^{*}(\phi)
$$

we first translate the fixed point $X^{*}(\phi)$ of system (5) to the origin of $\mathbb{R}^{6}$, and dropping tildes, we re-write the transformed system, which is also $K$-monotone, as

$$
X(t+1)=F(X(t)) .
$$

We claim that as long as $\phi^{*} \neq \phi^{\prime} \in(0,1)$, the origin is an asymptotically stable fixed point for (10) with respect to perturbations $\Delta X=(\Delta x, \Delta y)$ near $X=0$ for which $\Delta x$ is arbitrary, but for which $\Delta y \geq 0$. We shall first show that there are two vectors $u_{1} \leq_{K} 0$ and $0 \leq_{K} u_{2}$, such that the set $N=\left\{X \mid u_{1} \leq_{K} X \leq_{K} u_{2}\right\}$ is a compact neighborhood of $X=0$, and such that

$$
u_{1} \leq_{K} F\left(u_{1}\right) \leq_{K} 0 \leq_{K} F\left(u_{2}\right) \leq_{K} u_{2} .
$$


To establish this claim, we start by calculating the Jacobian matrix $J$ of $F(X)$ at $X=0$ :

$$
J=\left(\begin{array}{cc}
A_{1}\left(x^{*}\left(\phi^{*}\right), \phi^{*}\right)+B & B \\
0 & A_{1}\left(x^{*}\left(\phi^{*}\right), \phi^{\prime}\right)
\end{array}\right),
$$

where

$$
B=\left(\begin{array}{ccc}
0 & f_{M}^{\prime}\left(x_{M}^{*}\left(\phi^{*}\right)\right) x_{M}^{*}\left(\phi^{*}\right) & f_{N}^{\prime}\left(x_{N}^{*}\left(\phi^{*}\right)\right) x_{N}^{*}\left(\phi^{*}\right) \\
0 & 0 & 0 \\
0 & 0 & 0
\end{array}\right)
$$

The spectrum of $J$ consists of all the eigenvalues of the matrix $A_{1}\left(x^{*}\left(\phi^{*}\right), \phi^{*}\right)+B$ (all of which have modulus less than 1 , as shown in the proof of Theorem 2.1), and of the matrix $A_{1}\left(x^{*}\left(\phi^{*}\right), \phi^{\prime}\right)$, a nonnegative and irreducible (as $\left.\phi^{\prime} \in(0,1)\right)$ matrix whose dominant eigenvalue equals 1 , because by assumption $W\left(\phi^{*}, \phi^{\prime}\right)=0$, or equivalently by (6) because $R_{0}\left(x^{*}\left(\phi^{*}\right), \phi^{\prime}\right)=1$. Hence, the spectral radius of $J$ equals 1 as well. Now $J$ is a $K$-monotone matrix (meaning that $J$ maps the cone $K$ into itself), and thus the Perron-Frobenius Theorem for $K$-monotone matrices [43], implies that there is an eigenvector $0 \leq_{K} V$ corresponding to the dominant eigenvalue 1. In fact, in the Appendix we calculate $V$ explicitly, and show that $V$ belongs to the interior of $K$ for all $\phi^{\prime} \in(0,1)$. It is also shown in the Appendix that for all sufficiently small $\epsilon>0$, there holds that $u_{1} \leq_{K} F\left(u_{1}\right)$, when $u_{1}=-\epsilon V$.

The existence of a vector $0 \leq_{K} u_{2}$ such that $F\left(u_{2}\right) \leq_{K} u_{2}$ can be established as follows. We reconsider system (5) and assume that $\phi=\phi^{*}$, and that $\phi^{\prime} \in(0,1)$. Then the set $\left\{X=\left(\begin{array}{l}x \\ y\end{array}\right) \mid y=0\right\}$ is invariant, and the restriction of the dynamics to this invariant set, is such that all solutions with positive initial $x$-vector, converge to $x^{*}\left(\phi^{*}\right)$ by Theorem 2.1. Moreover, in Case 1 of the proof of Theorem 2.1, we have shown that there exists a positive vector $b$ in $\mathbb{R}^{3}$ such that $0 \leq T(b) \leq b$, where $T(x)=A_{1}\left(x, \phi^{*}\right) x$. In fact, $b$ can be chosen so that all its components are strictly larger than the corresponding components of $x^{*}\left(\phi^{*}\right)$. Consequently, by setting

$$
u_{2}=\left(\begin{array}{c}
b-x^{*}\left(\phi^{*}\right) \\
0
\end{array}\right)
$$

it follows that $u_{2} \geq_{K} 0$, and it can be verified that

$$
F\left(u_{2}\right)=\left(\begin{array}{c}
A_{1}\left(b, \phi^{*}\right) b-x^{*}\left(\phi^{*}\right) \\
0
\end{array}\right)=\left(\begin{array}{c}
T(b)-x^{*}\left(\phi^{*}\right) \\
0
\end{array}\right) \leq_{K}\left(\begin{array}{c}
b-x^{*}\left(\phi^{*}\right) \\
0
\end{array}\right)=u_{2}
$$

Combining the existence of the vectors $u_{1}$ and $u_{2}$ with the properties listed above, and the fact that system (10) is $K$-monotone, establishes (11). Notice in particular that the vectors $u_{1}$ and $u_{2}$ are such that $N$ is indeed a compact neighborhood of $X=0$.

Now, since system (10) is $K$-monotone, (11) implies that the orbit starting at $u_{1}$ is increasing with respect to the partial order $\leq_{K}$, and bounded above (by the zero fixed point). Thus, it must converge to some fixed point $X_{1}$ in $N$. Similarly, $K$-monotonicity and (11) imply that the orbit starting in $u_{2}$ is decreasing with respect to the partial order $\leq_{K}$, and bounded below by the zero fixed point, and must also converge to some fixed point $X_{2}$ in $N$. We now claim that we can always shrink $N$ by choosing $\epsilon>0$ sufficiently small in the definition of $u_{1}$, so that $X_{1}=X_{2}=0$. To see this, suppose that $(\tilde{x}, \tilde{y})$ is any fixed point of system (10) in $N$, with $\tilde{y} \geq 0$. If $\tilde{y}=0$, we first shrink $N$ by choosing $\epsilon>0$ sufficiently small in the definition of the vector $u_{1}$, so that $N$ does not include the fixed point $\left(-x^{*}\left(\phi^{*}\right), 0\right)$ of system $(10)$. Then $\tilde{x}$ must also be equal to 0 since $(0,0)$ is the unique fixed point in $N$ of system (10) restricted to the invariant set where $y=0$. Therefore, any fixed point $(\tilde{x}, \tilde{y})$ in $N$ is necessarily such that $\tilde{y} \neq 0$. In fact, the system equations (10) -see also (19) in the Appendix for the explicit functional forms- imply that $\tilde{y}$ must necessarily be a positive vector because $\phi^{\prime} \in(0,1)$. Moreover, as shown above, for any fixed point $(\tilde{x}, \tilde{y})$ in $N$, we have made sure that $\tilde{x}+x^{*}\left(\phi^{*}\right)$ is a positive vector as well. Therefore, if $N$ would contain a fixed point $(\tilde{x}, \tilde{y})$, other than $X=0$, then both $\tilde{x}+x^{*}\left(\phi^{*}\right)$ and $\tilde{y}$ would both be positive vectors. Then the original system (5) would have a positive fixed point as well. In the Appendix we show that this system can have at most one positive fixed point. By choosing $\epsilon>0$ even smaller in the definition of $u_{1}$, we can then ensure that the corresponding shifted fixed point for system (10), does not belong to $N$. Therefore, $N$ is an isolating neighborhood for the fixed point $X=0$, in the sense that it contains no other fixed points. In conclusion, we have proved the claim that $X_{1}=X_{2}=0$ by appropriately choosing $N$, and therefore by 
$K$-monotonicity, all solutions in the compact, invariant neighborhood $N$, converge to $X=0$. Going back to the original coordinates, we have proved that the fixed point $X^{*}\left(\phi^{*}\right)$ of the coupled system (5) is locally asymptotically stable, which completes the proof of the theorem.

Examples: We have shown earlier that the Beverton-Holt [2] and Hassell [14] fertility functions satisfy the two hypotheses (H1)-(H2). Next we show that they also satisfy the convexity condition $(\mathbf{C})$, from which follows that Theorem 3.4 applies when the fertility functions for migrants and non-migrants are of these types:

- The Beverton-Holt function [2]

$$
f_{B}(z)=\frac{a_{B}}{1+b_{B} z},
$$

where $a_{B}$ and $b_{B}$ are arbitrary positive constants, has the following second derivative:

$$
f_{B}^{\prime \prime}(z)=\frac{2 a_{B} b_{B}^{2}}{\left(1+b_{B} z\right)^{3}},
$$

which is positive for all $z \geq 0$, and thus $(\mathbf{C})$ holds.

- The Hassell function [14]:

$$
f_{H}(z)=\frac{a_{H}}{\left(1+b_{H} z\right)^{c_{H}}},
$$

where $a_{H}$ and $b_{H}$ are positive constants, and $0<c \leq 1$ (which is imposed to make (H1)-(H2) hold), has the following second derivative:

$$
f_{H}^{\prime \prime}=\frac{a_{H} b_{H}^{2} c_{H}\left(c_{H}+1\right)}{\left(1+b_{H} z\right)^{c_{H}+2}},
$$

which is positive for all $z \geq 0$, implying that $(\mathbf{C})$ holds.

3.4. Parametrizing models for salmonid species. Fecundity values of migrants and non-migrants vary widely by location. In most cases, migrants have greater fecundities than non-migrants, although in limited number of cases migrant fecundities are approximately equal to non-migrant. Individual fecundity is highly correlated with length [39, 3, 37] as well as age and the number of spawning migrations [31].

Mean non-migrant fecundities range from 170 eggs in Idaho desert populations [37], to 1400 eggs in a central Oregon population [38], and 3431 eggs in a western Alaska population [33], to 3065 eggs in an eastern Russian population [19].

Mean migrant fecundities range from 3438 in a coastal Oregon population [3], 3500 eggs in a central Oregon population [38], 4335-5706 eggs in a coastal Washington population [31], 7584 and 5171 in two California populations [36], and 10,638 in an eastern Russian population [19].

It is well documented among salmonids and their relatives (i.e., salmoniformes) that an increase in spawners leads to decreased spawning success and egg survival, therefore decreasing individual fertility $[42,11,26]$. To our knowledge, no fertility studies have been conducted directly on steelhead and rainbow trout, although they exhibit spawning behavior similar to many salmoniformes where density-dependent fertility taking the form of a Beverton-Holt function has been documented [12].

Survival probabilities also vary widely by location and are highly dependent on the age, size, and condition of an individual, as well as environmental conditions [35]. Migrants and no-nmigrants are indistinguishable as juveniles and therefore their early life survival is thought to be similar. Estimates derived from a number of sources indicate that survival from egg to ocean outmigrant or maturing non-migrant is 0.04 [30], although some estimates report juvenile annual survival at 0.0695 [25]. Survival to and from the ocean ranges from 0.03 to $0.17[39,46,45,30]$.

Based on these ranges of parameter values obtained from the literature, we now estimate the optimal allocation strategy $\phi^{*}$ for a specific example. We assume that migrants and non-migrants have BevertonHolt fertility functions:

$$
f_{M}(z)=\frac{8000}{1+\frac{z}{10}} \text { and } f_{N}(z)=\frac{1000}{1+\frac{z}{50}}
$$


given that migrant and non-migrant fecundities ranged from 3438 to 10638, and from 170 to 3431 respectively. We were unable to find estimates for the $b$-values in the above Beverton-Holt functions from the literature, but choose 1/10 for migrants and 1/50 for non-migrants. The ratio of five reflects that densitydependence is felt more quickly for migrants, i.e. for smaller abundance values, because of their larger size compared to non-migrants. Indeed, with this particular choice, the per capita fertility for migrants drops to half its maximum of 4000 for $z=10$, whereas the analogous drop for non-migrants occurs at an abundance of $z=50$, which is 5 times higher. Based on the above cited survival probabilities, we set:

$$
s_{M}=(0.04)(0.1)=0.004 \text { and } s_{N}=0.04 .
$$

These numbers are chosen because juvenile survival is similar for migrants and non-migrants with a probability of 0.04 , but migrants must survive their stay in the ocean which we assume occurs with probability 0.1 , given the range of 0.03 to 0.17 above. We also assume that non-migrants have a $100 \%$ survival rate in the river system in the same period of time, probably overestimating this rate somewhat. These parameter values are all we need to find the ESS value $\phi^{*}$. Indeed, first we note that with these values, we have that

$$
s_{M} f_{M}(0)=32, \text { and } s_{N} f_{N}(0)=40,
$$

both of which are larger than 1, which implies the existence of unique positive solutions to the equations $s_{M} f_{M}(z)=1$ and $s_{N} f_{N}(z)=1$. These are given by

$$
\hat{x}_{M}=10(32-1)=310 \text { and } \tilde{x}_{N}=50(40-1)=1950
$$

respectively. Recall also from Theorem 2.1 that $\hat{x}_{M}$ and $\tilde{x}_{N}$ are the respective fixed point abundances of migrants and non-migrants in populations that consist of migrants, respectively non-migrants only. Finally, we plug these values into (8) and obtain that

$$
\phi^{*}=\frac{310 / 1950}{310 / 1950+0.004 / 0.04}=94 \%,
$$

which means that the optimal allocation strategy is highly skewed towards migrants in this case.

\section{EvOlutionary DYNAMiCS}

An alternative to adaptive dynamics is to model evolution according to the Evolutionary Game Theory approach as advocated in $[20,21,44,1]$. In this methodology, an individual's allocation strategy is denoted by $v$, and the mean allocation strategy $\phi(t)$ in the population is treated as a dynamic state variable whose dynamics are governed by Lande's equation (or the breeder's equation, Fisher's equation, or the canonical equation of evolution). The methodology provides a coupled system for the population dynamics and the mean allocation strategy, known as the Darwinian dynamics:

$$
\begin{aligned}
\left(\begin{array}{c}
x_{1}(t+1) \\
x_{M}(t+1) \\
x_{N}(t+1)
\end{array}\right) & =\left.\left(\begin{array}{ccc}
0 & f_{M}\left(x_{M}(t)\right) & f_{N}\left(x_{N}(t)\right) \\
v s_{M} & 0 & 0 \\
(1-v) s_{N} & 0 & 0
\end{array}\right)\right|_{v=\phi(t)}\left(\begin{array}{c}
x_{1}(t) \\
x_{M}(t) \\
x_{N}(t)
\end{array}\right) \\
\phi(t+1) & =\phi(t)+\left.\sigma^{2} \frac{\partial \ln (\lambda(x(t), v)}{\partial v}\right|_{v=\phi(t)},
\end{aligned}
$$

Equation (14) states that the change in the mean strategy is proportional to the fitness gradient. Fitness here is taken to be $\ln (\lambda(x, v))$, where $\lambda(x, v)$ is the dominant eigenvalue of the matrix

$$
A_{1}(x, v)=\left(\begin{array}{ccc}
0 & f_{M}\left(x_{M}\right) & f_{N}\left(x_{N}\right) \\
v s_{M} & 0 & 0 \\
(1-v) s_{N} & 0 & 0
\end{array}\right) .
$$

The constant $\sigma^{2}$ is related to the (assumed constant) variance of the strategy throughout the population (equal, or proportional to the variance, depending on how the trait dynamics are derived) and is referred to as the speed of evolution.

A straightforward calculation shows that $\lambda(x, v)$ equals the square root of the basic reproduction number associated to $A_{1}(x, v)$, which we already defined in (3):

$$
\lambda(x, v)=\left(R_{0}(x, v)\right)^{1 / 2} \text {, where } R_{0}(x, v):=v s_{M} f_{M}\left(x_{M}\right)+(1-v) s_{N} f_{N}\left(x_{N}\right) .
$$


Hence, system (13) - (14) can be re-written as:

$$
\begin{aligned}
\left(\begin{array}{c}
x_{1}(t+1) \\
x_{M}(t+1) \\
x_{N}(t+1)
\end{array}\right) & =\left(\begin{array}{ccc}
0 & f_{M}\left(x_{M}(t)\right) & f_{N}\left(x_{N}(t)\right) \\
\phi(t) s_{M} & 0 & 0 \\
(1-\phi(t)) s_{N} & 0 & 0
\end{array}\right)\left(\begin{array}{c}
x_{1}(t) \\
x_{M}(t) \\
x_{N}(t)
\end{array}\right) \\
\phi(t+1) & =\phi(t)+\left.\frac{1}{2} \frac{\sigma^{2}}{R_{0}(x(t), \phi(t))} \frac{\partial R_{0}(x(t), v)}{\partial v}\right|_{v=\phi(t)},
\end{aligned}
$$

We first study this system for $\sigma=0$, i.e. when there are no evolutionary forces at work:

Theorem 4.1. Assume that $\sigma^{2}=0$. Suppose that (H1) - (H2) hold, and that (4) is satisfied. Then the following holds:

(1) For every fixed $\phi_{0}$ in $(0,1)$, every positive solution of system (15)-(16) with initial condition $\left(x_{0}, \phi_{0}\right)$ for arbitrary positive $x_{0}$, converges to a unique positive fixed point $\left(x^{*}\left(\phi_{0}\right), \phi_{0}\right)$, where $x^{*}\left(\phi_{0}\right)$ is the unique positive fixed point of system (1) with $\phi=\phi_{0}$ (see Theorem 2.1$)$. The fixed point $\left(x^{*}\left(\phi_{0}\right), \phi_{0}\right)$ is linearly stable with respect to positive initial conditions with arbitrary positive $x_{0}$, but fixed $\phi_{0}$.

(2) If $\phi_{0}=0$, then every positive solution of system (15) - (16) with initial condition $\left(x_{0}, \phi_{0}\right)$ for arbitrary positive $x_{0}$, converges to a unique non-zero fixed point $\left(\tilde{x}_{1}, 0, \tilde{x}_{N}, 0\right)$, where $\tilde{x}_{N}>0$ is the unique positive solution to the equation $s_{N} f_{N}(z)=1$, and $\tilde{x}_{1}=\tilde{x}_{N} / s_{N}$. This fixed point is linearly stable with respect to initial conditions with arbitrary positive $x_{0}$, but fixed $\phi_{0}=1$.

(3) If $\phi_{0}=1$, then every positive solution of system (15)-(16) with initial condition $\left(x_{0}, 0\right)$ for arbitrary positive $x_{0}$, converges to a unique non-zero fixed point $\left(\hat{x}_{1}, \hat{x}_{M}, 0,1\right)$, where $\tilde{x}_{N}>0$ is the unique positive solution to the equation $s_{M} f_{M}(z)=1$, and $\hat{x}_{1}=\hat{x}_{M} / s_{M}$. This fixed point is linearly stable with respect to initial conditions with arbitrary positive $x_{0}$, but fixed $\phi_{0}=0$.

The function $\phi \rightarrow x^{*}(\phi)$ is smooth, and $\lim _{\phi \rightarrow 0} x^{*}(\phi)=\left(\tilde{x}_{1}, 0, \tilde{x}_{N}\right)$ and $\lim _{\phi \rightarrow 1} x^{*}(\phi)=\left(\hat{x}_{1}, \hat{x}_{M}, 0\right)$.

Proof. The proof follows immediately from Theorem 2.1, and the fact that for each $\phi_{0}$ in $[0,1]$, the set $\left\{(x, \phi) \in \mathbb{R}_{+}^{3} \times[0,1] \mid \phi=\phi_{0}\right\}$ is forward invariant for solutions of system (15) - (16) when $\sigma^{2}=0$.

We now turn to the case where $\sigma^{2}>0$. In addition to the assumptions made in Theorem 4.1, we impose the following condition which is generically satisfied:

$$
s_{M} f_{M}(0) \neq s_{N} f_{N}(0) \text {. }
$$

Equivalently, $R_{0}(0, \phi)=\phi s_{M} f_{M}(0)+(1-\phi) s_{N} f_{N}(0)$, considered as a function of the variable $\phi$ with values in $[0,1]$, should not be a constant function.

In the appendix, we prove the following result.

Theorem 4.2. Assume that $\sigma^{2}>0$. Suppose that $(\mathbf{H 1})-(\mathbf{H 2})$ hold, and that (4) and (17) are satisfied.

For all $\sigma^{2}>0$, system $(15)-(16)$ has a unique fixed point $\left(x^{*}\left(\phi^{*}\right), \phi^{*}\right)$ in $\mathbb{R}_{+}^{3} \times[0,1]$, where $\phi^{*}$ is given by formula (8), and $x^{*}\left(\phi^{*}\right)$ is the unique positive fixed point of system (1) with $\phi=\phi^{*}$ (see Theorem 2.1). Moreover, there exists $\sigma_{*}^{2}>0$, such that $\left(x^{*}\left(\phi^{*}\right), \phi^{*}\right)$ is a locally asymptotically stable fixed point of system (15) - (16) for all $0<\sigma^{2}<\sigma_{*}^{2}$.

\section{Comparing adaptive dynamics And evolutionary game theoretic approaches}

The goal of this paper was to explain how partial migration has evolved, first using an adaptive dynamics framework which led to Theorem 3.4, and secondly, by applying the canonical model of evolutionary game theory which resulted in Theorem 4.2. Perhaps the most striking conclusion is that both approaches lead to the same, unique evolutionary stable allocation strategy value $\phi^{*}$ (for every individual in the context of adaptive dynamics, but only for the population mean in the evolutionary game theory methodology ${ }^{1}$,

\footnotetext{
${ }^{1}$ In the context of evolutionary game theory, a mean population strategy $\phi^{*}$ is said to be an ESS if the Darwinian dynamics has an asymptotically stable fixed point $\left(x^{*}, \phi^{*}\right)$, and is such that $\lambda\left(x^{*}, v\right)$, seen as a function of the variable $v$, has a global maximum at $v=\phi^{*}$. Note that here, $\lambda\left(x^{*}, v\right)$ is a constant function of $v$, and equal to 1 ; for further discussion of the ESS concept in evolutionary game theory, see [44].
} 
given by the formula (8), and reproduced here:

$$
\phi^{*}=\frac{\hat{x}_{M} / \tilde{x}_{N}}{\hat{x}_{M} / \tilde{x}_{N}+s_{M} / s_{N}} .
$$

Here, $\hat{x}_{M}$ and $\tilde{x}_{N}$ are the unique roots of the equation $s_{M} f_{M}(z)=1$ and $s_{N} f_{N}(z)=1$ respectively, see Theorem 2.1. These roots represent the fixed point level of migrant adults for a model where no eggs become non-migrants (model (1) with $\phi=1$ ), respectively the fixed point level of non-migrant adults for a model where no eggs grow into migrants (model (1) with $\phi=0$ ). This formula can be used to predict the optimal allocation strategy towards migrants, when the vital parameters of both subtypes are known.

The main difference between both approaches is that we require the convexity condition $(\mathbf{C})$ in the proof that $\phi^{*}$ is an ESS of the adaptive dynamics. Condition $(\mathbf{C})$ expresses that the negative density dependence in the fertility functions $f_{M}$ and $f_{N}$ should be attenuated. The result based on evolutionary game theory in Theorem 4.2 does not require such a condition. This suggests that perhaps condition (C) may not be necessary in the adaptive dynamics setting. We remark that condition $(\mathbf{C})$ is only a sufficient condition used to establish that $\phi^{*}$ is an ESS for the adaptive dynamics; currently, we do not know whether it is also a necessary condition.

\section{Conclusions}

This work concisely demonstrates that partial migration can be attributed to density dependence alone, and does not have to rely on strategy dependence (also known as "frequency dependence" [24]), or other features. The density dependence used in this model is perhaps the simplest form of density dependence wherein the densities of migrants and non-migrants only affect a vital rate within their own type.

There are several possible extensions of the underlying population model (1). For example, we have ignored juveniles, by only modeling eggs and adults. It is at least conceivable that the introduction of juvenile classes whose abundances negatively affect adult fertilities, will not necessarily lead to an ESS with a corresponding locally stable fixed point $X^{*}\left(\phi^{*}\right)$ for the coupled model (5), but instead exhibits a stable synchronous periodic cycle. Further work is needed to examine this possibility.

Another important feature of the population model (1) is that it assumes that the fertilities of migrants and non-migrants is not negatively affected by the other subtype. Although this assumption is reasonable for O. mykiss, it is not valid for other species exhibiting partial migration, with other forms of density dependence $[18,41,13]$. For example, in some bird populations, nonmigrants may only experience density dependence in fertilities caused by other nonmigrant individuals, whereas migrants experience competitive effects caused by both migrants and non-migrants alike. Indeed, nonmigrants will be able to select the best breeding grounds prior to the return of the migrants. The latter will have to deal with nonmigrants that have already established a breeding spot, and with other returning migrants. Mathematically, scenarios like this one, can be described by replacing $f_{M}\left(x_{M}(t)\right)$ in model (1) by $f_{M}\left(x_{M}(t)+x_{N}(t)\right)$, but retaining all other model parameters and functionals. Although this operation may be perceived as relatively minor, it is by no means harmless: the resulting population model is no longer monotone, and therefore alternative proof techniques will have to be developed to understand the behavior of these models. Nevertheless, our results can serve as a starting point and background to compare the behavior of such models to.

\section{ACKNOWLEDGMENTS}

PDL acknowledges partial support from NSF-DMS-1411853. This work was funded by Bonneville Power Administration (BPA) project 2003-017 to HAO and DAL, with additional support provided by GH Reeves. 


\section{Appendix}

\subsection{Proof of Theorem 2.1.}

Proof. Case 1: $\phi \in(0,1)$.

We first show that for each $\phi \in(0,1)$, there exists a positive vector positive vector $b$, such that the set:

$$
[0, b]:=\left\{z \in \mathbb{R}_{+}^{3} \mid 0 \leq x \leq b\right\},
$$

is compact, forward invariant, and absorbing for system (1). To see this, notice first that continuity of $R_{0}(x, \phi)$, as well as condition (4), imply the existence of a positive vector $a_{\phi}$ such that $R_{0}\left(a_{\phi}, \phi\right)<1$. Consequently, the dominant eigenvalue of the non-negative matrix $A_{1}\left(a_{\phi}, \phi\right)$, denoted by $\lambda_{P}$, is also strictly less than 1 by the results in [22]. Notice that the matrix $A_{1}\left(a_{\phi}, \phi\right)$ is irreducible, hence by the PerronFrobenius Theorem there is a positive eigenvector $z_{\phi}$ corresponding to the dominant eigenvalue $\lambda_{P}$ :

$$
A_{1}\left(a_{\phi}, \phi\right) z_{\phi}=\lambda_{P} z_{\phi}
$$

We assume without loss of generality that $\left\|z_{\phi}\right\|=1$. Let $\gamma^{*}>0$ be such that for all $\gamma \geq \gamma^{*}$ :

$$
a_{\phi} \leq \gamma z_{\phi}
$$

Such a $\gamma^{*}$ clearly exists because $z_{\phi}$ is a positive vector. Condition $(\mathbf{H 1})$ then implies that for all $\gamma \geq \gamma^{*}$ :

$$
A_{1}\left(\gamma z_{\phi}, \phi\right) \leq A_{1}\left(a_{\phi}, \phi\right),
$$

where the former inequality holds entry-wise for both matrices. This implies that for all $\gamma \geq \gamma^{*}$ :

$$
A_{1}\left(\gamma z_{\phi}, \phi\right)\left(\gamma z_{\phi}\right) \leq A_{1}\left(a_{\phi}, \phi\right)\left(\gamma z_{\phi}\right)=\lambda_{P}\left(\gamma z_{\phi}\right) .
$$

Thus, by setting $b:=\gamma^{*} z_{\phi}$, and using the monotonicity of the system, the latter shows that $[0, b]$ is forward invariant: If $0 \leq x \leq b$, then $0=T(0) \leq T(x) \leq T(b) \leq \lambda_{P} b \leq b$, because $\lambda_{P}<1$. The set $[0, b]$ is clearly compact and we have just established that it is forward invariant. To show that it is absorbing, let $y \geq 0$ be an arbitrary state. Then there is a $\gamma_{y}>\gamma^{*}$ such that:

$$
y \leq \gamma_{y} z_{\phi}
$$

Monotonicity and (18) imply that

$$
0=T(0) \leq T(y) \leq T\left(\gamma_{y} z_{\phi}\right) \leq \lambda_{P}\left(\gamma_{y} z_{\phi}\right),
$$

and more generally that:

$$
0 \leq T^{r}(y) \leq \lambda_{P}^{r}\left(\gamma_{y} z_{\phi}\right)
$$

for all positive integers $r$ for which $\lambda_{P}^{r-1} \gamma_{y}>\gamma^{*}$. Since $\lambda_{P}<1$, there exists a minimal positive integer $r^{*}$ such that $\lambda_{P}^{r^{*}} \gamma_{y} \leq \gamma^{*}$, and hence that

$$
0 \leq T^{r^{*}}(y) \leq \lambda_{P}^{r^{*}}\left(\gamma_{y} z_{\phi}\right) \leq \gamma^{*} z_{\phi}=b
$$

which shows that the orbit starting in $y$ is absorbed in the set $[0, b]$ in the $r^{*}$ th step.

Next, we establish that there exists a positive fixed point. First, the right inequality in (4) implies that the zero fixed point is linearly unstable by the results in [22]. Note that the linearization of (1) at the zero fixed point is given by $A_{1}(0, \phi)$, which is a non-negative, irreducible matrix. Hence, this linearization has a dominant eigenvalue $\mu>1$, and corresponding positive eigenvector $v$ :

$$
A_{1}(0, \phi) v=\mu v
$$

We shall consider the orbit starting in sufficiently small positive scalar multiples of the vector $v$, and show that these orbits must be increasing. A Taylor expansion of $T$ yields that

$$
T(\epsilon v)=T(0)+A_{1}(0, \phi)(\epsilon v)+o(\epsilon)=\mu(\epsilon v)+o(\epsilon) \geq \epsilon v,
$$

where the last inequality holds for all sufficiently small and positive $\epsilon$ because $\mu>1$. Exploiting monotonicity, we see that the orbit starting in $\epsilon v$ is indeed increasing. Since it is bounded above (by $b$ ), it must converge, and the limit is necessarily a fixed point, which we denote by $x_{1}^{*}$. Then $x_{1}^{*}$ is clearly positive since $x_{1}^{*} \geq \epsilon v$, and $\epsilon v$ is a positive vector. We have already shown that $T(b) \leq b$, and exploiting monotonicity 
once again, we see that the orbit starting in $b$ is decreasing. Since it is bounded below (by 0), it converges to a fixed point, denoted by $x_{2}^{*}$. In fact, we have that:

$$
0=T(0) \leq \epsilon v \leq T(\epsilon v) \leq T(b) \leq b,
$$

and by monotonicity, there follows that:

$$
x_{1}^{*} \leq x_{2}^{*}
$$

We claim that in fact

$$
x_{1}^{*}=x_{2}^{*} .
$$

To see this, note that since $x_{1}^{*}$ and $x_{2}^{*}$ are fixed points, there holds that:

$$
A_{1}\left(x_{1}^{*}, \phi\right) x_{1}^{*}=x_{1}^{*} \text { and } A_{1}\left(x_{2}^{*}, \phi\right) x_{2}^{*}=x_{2}^{*}
$$

Since both above matrices are non-negative and irreducible, and the vectors $x_{1}^{*}$ and $x_{2}^{*}$ are positive vectors, the Perron-Frobenius Theorem implies that their respective dominant eigenvalues $\lambda_{1}$ and $\lambda_{2}$ are both equal to 1 . However, we also have that

$$
A_{1}\left(x_{1}^{*}, \phi\right) \leq A_{1}\left(x_{2}^{*}, \phi\right) \text { and } A_{1}\left(x_{1}^{*}, \phi\right) \neq A_{1}\left(x_{2}^{*}, \phi\right),
$$

because of condition (H1) (as before, the inequality between the above two matrices holds entry-wise). But then the Perron-Frobenius Theorem would imply that $\lambda_{1}<\lambda_{2}$, contradicting that $\lambda_{1}=\lambda_{2}=1$. Henceforth, we denote $x_{1}^{*}$ as $x^{*}(\phi)$, to emphasize that the fixed point depends on $\phi$.

We claim that the orbit of every positive initial condition $x_{0}$ in $[0, b]$, converges to $x^{*}(\phi)$. To see this, note that there is some $\epsilon>0$ such that $\epsilon v \leq x_{0} \leq b$. Exploiting monotonicity, and the fact that the orbits starting in $\epsilon v$ and $b$ converge to $x^{*}(\phi)$, it follows that the orbit starting in $x_{0}$ converges to $x^{*}(\phi)$ as well. In particular, $x^{*}(\phi)$ is the unique, positive fixed point in $[0, b]$. Finally, since $[0, b]$ is absorbing, every positive orbit eventually enters $[0, b]$, and the first time it enters $[0, b]$, this occurs in some positive vector. Therefore, every orbit starting in a positive vector must converge to $x^{*}(\phi)$ as well. To see that $x^{*}(\phi)$ is linearly stable, consider the Jacobian matrix at $x^{*}(\phi)$ :

$$
J\left(x^{*}(\phi)\right)=A_{1}\left(x^{*}(\phi), \phi\right)+\left(\begin{array}{ccc}
0 & - & - \\
0 & 0 & 0 \\
0 & 0 & 0
\end{array}\right)
$$

where the -signs in the matrix above represent negative values in view of condition (H1), although their actual values are irrelevant for what values. Therefore,

$$
J\left(x^{*}(\phi)\right) \leq A_{1}\left(x^{*}(\phi), \phi\right) \text {, and } J\left(x^{*}(\phi)\right) \neq A_{1}\left(x^{*}(\phi), \phi\right) .
$$

Since the dominant eigenvalue of $A_{1}\left(x^{*}(\phi), \phi\right)$ equals 1 , as we established above, the Perron-Frobenius Theorem implies that the dominant eigenvalue of the non-negative and irreducible matrix $J\left(x^{*}(\phi)\right)$ is less than 1. This implies the linear stability of the fixed point $x^{*}(\phi)$.

Case 2: $\phi=0$.

First notice that every orbit of (1) enters the invariant part of the the boundary of $\mathbb{R}_{+}^{3}$ where $x_{M}=0$ in 1 step. The restriction of the dynamics to this part of the boundary is given by a planar and monotone system:

$$
\left(\begin{array}{c}
x_{1}(t+1) \\
x_{N}(t+1)
\end{array}\right)=\left(\begin{array}{cc}
0 & f_{N}\left(x_{N}(t)\right) \\
s_{N} & 0
\end{array}\right)\left(\begin{array}{c}
x_{1}(t) \\
x_{N}(t)
\end{array}\right)
$$

Assumption (4) with $\phi=0$ implies that the zero fixed point of the above system is linearly unstable, and that it has a compact, invariant and absorbing set of the form $\left[0, b_{2}\right]$ for some positive vector $b_{2}$. This can be proved using similar arguments we used to establish a comparable result for system (1) in the case where $\phi \in(0,1)$. Along the same lines, it can also be proved that there is a unique positive fixed point in $\left[0, b_{2}\right]$, which is both locally stable, as well as globally attractive for all positive orbits. In fact, it is straightforward to calculate this fixed point explicitly; it equals $\left(\tilde{x}_{N} / s_{N}, \tilde{x}_{N}\right)$, where $\tilde{x}_{N}$ is the unique solution to the equation $s_{N} f_{N}(z)=1$. (Note that condition (4) can be rephrased as $s_{N} f_{N, \infty}<1<s_{N} f_{N}(0)$ ), and since $f_{N}(z)$ is decreasing by condition $(\mathbf{H 1})$, there must be a unique solution $\left.\tilde{x}_{N}\right)$. Linear stability of the corresponding fixed point $\left(\tilde{x}_{N} / s_{N}, 0, \tilde{x}_{N}\right)$ of system (1) with $\phi=0$ can be established using a linearization argument as before. And global convergence of positive orbits to this fixed point follows because the fixed 
point $\left(\tilde{x}_{N} / s_{N}, \tilde{x}_{N}\right)$ attracts all positive orbits of the planar system above, in conjunction with the fact that all orbits of system (1) enter the part of the boundary where $x_{M}=0$ in 1 step.

Case 3: $\phi=1$.

The proof is similar to the case $\phi=0$.

To conclude the proof, we remark that the smoothness of the fixed point $x^{*}(\phi)$ when $\phi$ belongs to $[0,1]$ follows from the Implicit Function Theorem, applied to the fixed point equation $x=A_{1}(x, \phi) x$, and the fact established above that the Jacobian matrix $J\left(x^{*}(\phi)\right)$ has dominant eigenvalue less than 1 . The statements regarding the limiting values of $x^{*}(\phi)$ as $\phi$ approaches 0 or 1 , follow from the uniqueness of the positive fixed point (when $\phi \in(0,1)$ ), and of the non-negative fixed point (when $\phi=0$ or 1 ).

8.2. Dynamics along a dominant eigenvector. Recall system (10), where the map $F(X)$ is given explicitly by:

$$
\left(\begin{array}{c}
f_{M}\left(x_{M}+y_{M}+x_{M}^{*}\left(\phi^{*}\right)\right)\left(x_{M}+x_{M}^{*}\left(\phi^{*}\right)\right)+f_{N}\left(x_{N}+y_{N}+x_{N}^{*}\left(\phi^{*}\right)\right)\left(x_{N}+x_{N}^{*}\left(\phi^{*}\right)\right)-x_{1}^{*}\left(\phi^{*}\right) \\
s_{M} \phi^{*}\left(x_{1}+x_{1}^{*}\left(\phi^{*}\right)\right)-x_{M}^{*}\left(\phi^{*}\right) \\
s_{N}\left(1-\phi^{*}\right)\left(x_{1}+x_{1}^{*}\left(\phi^{*}\right)\right)-x_{N}^{*}\left(\phi^{*}\right) \\
y_{M} f_{M}\left(x_{M}+y_{M}+x_{M}^{*}\left(\phi^{*}\right)\right)+y_{N} f_{N}\left(x_{N}+y_{N}+x_{N}^{*}\left(\phi^{*}\right)\right) \\
s_{M} \phi^{\prime} y_{1} \\
s_{N}\left(1-\phi^{\prime}\right) y_{1}
\end{array}\right)
$$

Let us start by determining an eigenvector $0 \leq_{K} V$ corresponding to the dominant eigenvalue 1 of the Jacobian matrix $J$, obtained by linearizing $F$ at $X=0$, and given in (12). There must hold that $J V=V$, or more explicitly, by denoting $V=\left(\begin{array}{c}v \\ \tilde{v}\end{array}\right)=\left(v_{1}, v_{2}, v_{3}, \tilde{v}_{1}, \tilde{v}_{2}, \tilde{v}_{3}\right)^{T}$, that:

$$
\begin{aligned}
A_{1}\left(x^{*}\left(\phi^{*}\right), \phi^{\prime}\right) \tilde{v} & =\tilde{v} \\
\left(A_{1}\left(x^{*}\left(\phi^{*}\right), \phi^{*}\right)+B\right) v+B \tilde{v} & =v,
\end{aligned}
$$

where

$$
A_{1}\left(x^{*}\left(\phi^{*}\right), \phi^{\prime}\right)=\left(\begin{array}{ccc}
0 & 1 / s_{M} & 1 / s_{N} \\
s_{M} \phi^{\prime} & 0 & 0 \\
s_{N}\left(1-\phi^{\prime}\right) & 0 & 0
\end{array}\right)
$$

and

$$
A_{1}\left(x^{*}\left(\phi^{*}\right), \phi^{*}\right)+B=\left(\begin{array}{ccc}
0 & 1 / s_{M}+f_{M}^{\prime}\left(x_{M}^{*}\left(\phi^{*}\right)\right) x_{M}^{*}\left(\phi^{*}\right) & 1 / s_{N}+f_{N}^{\prime}\left(x_{N}^{*}\left(\phi^{*}\right)\right) x_{N}^{*}\left(\phi^{*}\right) \\
s_{M} \phi^{*} & 0 & 0 \\
s_{N}\left(1-\phi^{*}\right) & 0 & 0
\end{array}\right) .
$$

We first find a nonzero solution $\tilde{v}$ for (20). This must be a non-positive vector because we're looking for an eigenvector $V$ in the cone $K$ :

$$
\tilde{v}=\left(\begin{array}{c}
-1 \\
-s_{M} \phi^{\prime} \\
-s_{N}\left(1-\phi^{\prime}\right)
\end{array}\right)
$$

Plugging this into (21), and rearranging yields that $v$ must satisfy:

$$
\left(\begin{array}{ccc}
-1 & \frac{1}{s_{M}}+f_{M}^{\prime}\left(x_{M}^{*}\left(\phi^{*}\right)\right) x_{M}^{*}\left(\phi^{*}\right) & \frac{1}{s_{N}}+f_{N}^{\prime}\left(x_{N}^{*}\left(\phi^{*}\right)\right) x_{N}^{*}\left(\phi^{*}\right) \\
s_{M} \phi^{*} & -1 & 0 \\
s_{N}\left(1-\phi^{*}\right) & 0 & -1
\end{array}\right) v=\left(\begin{array}{l}
b \\
0 \\
0
\end{array}\right)
$$

where $b=f_{M}^{\prime}\left(x_{M}^{*}\left(\phi^{*}\right)\right) x_{M}^{*}\left(\phi^{*}\right) s_{M} \phi^{\prime}+f_{N}^{\prime}\left(x_{N}^{*}\left(\phi^{*}\right)\right) x_{N}^{*}\left(\phi^{*}\right) s_{N}\left(1-\phi^{\prime}\right)<0$ because of (H1). Solving (22) for the vector $v$ yields:

$$
v=\frac{1}{\operatorname{det}}\left(\begin{array}{c}
b \\
s_{M} \phi^{*} b \\
s_{N}\left(1-\phi^{*}\right) b
\end{array}\right), \text { where } \operatorname{det}=\phi^{*} s_{M} f_{M}^{\prime}\left(x_{M}^{*}\left(\phi^{*}\right)\right) x_{M}^{*}\left(\phi^{*}\right)+\left(1-\phi^{*}\right) s_{N} f_{N}^{\prime}\left(x_{N}^{*}\left(\phi^{*}\right)\right) x_{N}^{*}\left(\phi^{*}\right)<0,
$$


again because of $(\mathbf{H 1})$. The vector $v$ is a positive vector, as expected. In summary, an eigenvector $0 \leq_{K} V$ for the dominant eigenvalue 1 of the matrix $J$ is given by:

$$
V=\left(\begin{array}{c}
b / \operatorname{det} \\
s_{M} \phi^{*} b / \operatorname{det} \\
s_{N}\left(1-\phi^{*}\right) b / \operatorname{det} \\
-1 \\
-s_{M} \phi^{\prime} \\
-s_{N}\left(1-\phi^{\prime}\right)
\end{array}\right)
$$

Note also that $V$ belongs to the interior of $K$ because $\phi^{\prime} \in(0,1)$. Next, for any $\epsilon>0$, we set

$$
u_{1}=-\epsilon V=\epsilon\left(\begin{array}{c}
-b / \text { det } \\
-s_{M} \phi^{*} b / \text { det } \\
-s_{N}\left(1-\phi^{*}\right) b / \text { det } \\
1 \\
s_{M} \phi^{\prime} \\
s_{N}\left(1-\phi^{\prime}\right)
\end{array}\right) \text {, }
$$

and therefore

$$
u_{1} \leq_{K} 0 .
$$

We now proceed to show that for all sufficiently small $\epsilon>0$, there also holds that:

$$
u_{1} \leq_{K} F\left(u_{1}\right) \text {. }
$$

By using a Taylor expansion for $F$ near $X=0$,

$$
F\left(u_{1}\right)=J u_{1}+h\left(u_{1}\right)+O\left(\left\|u_{1}\right\|^{3}\right)=u_{1}+h\left(u_{1}\right)+O\left(\left\|u_{1}\right\|^{3}\right) .
$$

Here, $h=\left(h_{1}, h_{2}, h_{3}, h_{4}, h_{5}, h_{6}\right)^{T}$ with each $h_{i}\left(u_{1}\right)=u_{1}^{T} H_{i} u_{1}$ and $H_{i}$ being the Hessian with respect to $F_{i}$. One can verify that $h_{2}=h_{3}=h_{5}=h_{6}=0$ and that the corresponding higher order terms $O_{2}=O_{3}=O_{5}=O_{6}$ vanish too because $F_{2}, F_{3}, F_{5}$ and $F_{6}$ are affine functions in $X$. Henceforth, we focus on finding the sign of $h_{1}\left(u_{1}\right)$ and $h_{4}\left(u_{1}\right)$.

We start by calculating $h_{1}\left(u_{1}\right)=u_{1}^{T} H_{1} u_{1}$ where $H_{1}$ is the Hessian corresponding to $F_{1}$, where from now on we drop the argument $\phi^{*}$ from the functions $x_{M}^{*}\left(\phi^{*}\right)$ and $x_{N}^{*}\left(\phi^{*}\right)$ to economize on the notation:

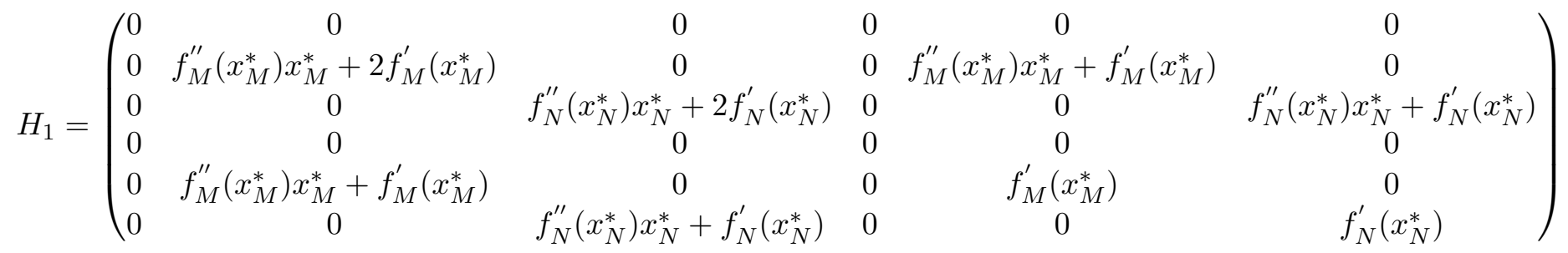

We denote $u_{1}=\left(u_{1_{1}}, u_{1_{2}}, u_{1_{3}}, u_{1_{4}}, u_{1_{5}}, u_{1_{6}}\right)^{T}$, and simplify $h_{1}\left(u_{1}\right)$ to:

$$
h_{1}\left(u_{1}\right)=(c-\alpha)\left(u_{1_{2}}+u_{1_{5}}\right)^{2}+(d-\beta)\left(u_{1_{3}}+u_{1_{6}}\right)^{2}+\alpha u_{1_{5}}\left(u_{1_{2}}+u_{1_{5}}\right)+\beta u_{1_{6}}\left(u_{1_{3}}+u_{1_{6}}\right),
$$

where $c=f_{M}^{\prime \prime}\left(x_{M}^{*}\right) x_{M}^{*}, d=f_{N}^{\prime \prime}\left(x_{N}^{*}\right) x_{N}^{*}, \alpha=-2 f_{M}^{\prime}\left(x_{M}^{*}\right)$ and $\beta=-2 f_{N}^{\prime}\left(x_{N}^{*}\right)$. Our goal is te re-write $h_{1}\left(u_{1}\right)$ as a function of the variable $z=\phi^{*}-\phi^{\prime}$. The coefficients $c, d, \alpha$ and $\beta$ only depend on $\phi^{*}$, but not on $\phi^{\prime}$. Moreover, we can express $\left(u_{1_{2}}+u_{1_{5}}\right),\left(u_{1_{3}}+u_{1_{6}}\right), u_{1_{5}}$ and $u_{1_{6}}$ in terms of $z$ using the various values of the components of $u_{1}$ from (23):

$$
\begin{aligned}
u_{1_{2}}+u_{1_{5}} & =-\frac{\epsilon}{d e t} f_{N}^{\prime}\left(x_{N}^{*}\right) x_{N}^{*} s_{M} s_{N} z=:-\epsilon C_{1}\left(\phi^{*}\right) z \\
u_{1_{3}}+u_{1_{6}} & =\frac{\epsilon}{\operatorname{det}} f_{M}^{\prime}\left(x_{M}^{*}\right) x_{M}^{*} s_{M} s_{N} z=: \epsilon C_{2}\left(\phi^{*}\right) z \\
u_{1_{5}} & =\epsilon\left(-s_{M} z+s_{M} \phi^{*}\right) \\
u_{1_{6}} & =\epsilon\left(s_{N}(1+z)-s_{N} \phi^{*}\right)
\end{aligned}
$$


By plugging $(27)-(30)$ into $(26)$, we obtain $h_{1}\left(u_{1}\right) / \epsilon^{2}$ as a function of $z$, denoted by $Q_{1}(z)$ :

$$
\begin{aligned}
Q_{1}(z)= & {\left[(c-\alpha) C_{1}^{2}\left(\phi^{*}\right)+(d-\beta) C_{2}^{2}\left(\phi^{*}\right)+\alpha s_{M} C_{1}\left(\phi^{*}\right)+\beta s_{N} C_{2}\left(\phi^{*}\right)\right] z^{2}+} \\
& {\left[-\alpha s_{M} \phi^{*} C_{1}\left(\phi^{*}\right)+\beta s_{N}\left(1-\phi^{*}\right) C_{2}\left(\phi^{*}\right)\right] z }
\end{aligned}
$$

Clearly, $Q_{1}(0)=0$,

$$
\begin{aligned}
Q_{1}^{\prime}(0) & =-\alpha s_{M} \phi^{*} C_{1}\left(\phi^{*}\right)+\beta s_{N}\left(1-\phi^{*}\right) C_{2}\left(\phi^{*}\right) \\
& =\frac{2}{d e t} s_{M} s_{N} f_{M}^{\prime}\left(x_{M}^{*}\right) f_{N}^{\prime}\left(x_{N}^{*}\right)\left[s_{M} \phi^{*} x_{M}^{*}-s_{N}\left(1-\phi^{*}\right) x_{N}^{*}\right] \\
& =0,
\end{aligned}
$$

were we have used that $x_{M}^{*}=s_{M} \phi^{*} x_{1}^{*}$ and that $x_{N}^{*}=s_{N}\left(1-\phi^{*}\right) x_{1}^{*}$. These latter two equalities can also be used to show that:

$$
\begin{aligned}
Q_{1}^{\prime \prime}(0) & =\frac{2}{\operatorname{det}^{2}}\left[\left(f_{N}^{\prime}\left(x_{N}^{*}\right) x_{N}^{*} s_{M} s_{N}\right)^{2} f_{M}^{\prime \prime}\left(x_{M}^{*}\right) x_{M}^{*}+\left(f_{M}^{\prime}\left(x_{M}^{*}\right) x_{M}^{*} s_{M} s_{N}\right)^{2} f_{N}^{\prime \prime}\left(x_{N}^{*}\right) x_{N}^{*}\right] \\
& >0,
\end{aligned}
$$

where we have used the convexity assumption that $f_{N}^{\prime \prime}\left(x_{N}^{*}\right)>0$ and $f_{M}^{\prime \prime}\left(x_{M}^{*}\right)>0$. This implies that $Q_{1}(z)>0$ for all $z \neq 0$, and thus that $h_{1}\left(u_{1}\right)>0$ for all $\phi^{\prime} \neq \phi^{*}$.

Similarly, $h_{4}\left(u_{1}\right)=u_{1}^{T} H_{4} u_{1}$, where the Hessian $H_{4}$ takes the form

$$
H_{4}=\left(\begin{array}{cccccc}
0 & 0 & 0 & 0 & 0 & 0 \\
0 & 0 & 0 & 0 & p & 0 \\
0 & 0 & 0 & 0 & 0 & q \\
0 & 0 & 0 & 0 & 0 & 0 \\
0 & p & 0 & 0 & 2 p & 0 \\
0 & 0 & q & 0 & 0 & 2 q
\end{array}\right) \text {, with } p=f_{M}^{\prime}\left(x_{M}^{*}\right) \text { and } q=f_{N}^{\prime}\left(x_{N}^{*}\right)
$$

Therefore,

$$
h_{4}\left(u_{1}\right)=2 p u_{1_{5}}\left(u_{1_{2}}+u_{1_{5}}\right)+2 q u_{1_{6}}\left(u_{1_{3}}+u_{1_{6}}\right)
$$

By plugging $(27)-(30)$ into $(31)$, and setting $Q_{4}(z)=h_{4}\left(u_{1}\right) / \epsilon^{2}$, we obtain

$$
Q_{4}(z)=\left[2 p s_{M} C_{1}\left(\phi^{*}\right)+2 q s_{N} C_{2}\left(\phi^{*}\right)\right] z^{2}+\left[2 q s_{N} C_{2}\left(\phi^{*}\right)\left(1-\phi^{*}\right)-2 p s_{M} C_{1}\left(\phi^{*}\right) \phi^{*}\right] z
$$

It can be verified that $Q_{4}(0)=0=Q_{4}^{\prime}(0)$, and that

$$
\begin{aligned}
Q_{4}^{\prime \prime}(0) & =\frac{\left.4 s_{M} s_{N} f_{M}^{\prime}\left(x_{M}^{*}\right) f_{N}^{\prime}\left(x_{N}^{*}\right)\right)}{\operatorname{det}}\left(s_{M} x_{N}^{*}+s_{N} x_{M}^{*}\right) \\
& <0 .
\end{aligned}
$$

This implies that $Q_{4}(z)<0$ for all $z \neq 0$, and thus that $h_{4}\left(u_{1}\right)<0$ for all $\phi^{\prime} \neq \phi^{*}$. Consequently, (24) holds for all sufficiently small $\epsilon>0$.

8.3. At most one positive fixed point. We will show that the coupled system (5) has at most one positive fixed point when $\phi=\phi^{*}$ and $\phi^{\prime} \neq \phi^{*}$. First recall the system equations:

$$
\left(\begin{array}{c}
x_{1}(t+1) \\
x_{M}(t+1) \\
x_{N}(t+1) \\
y_{1}(t+1) \\
y_{M}(t+1) \\
y_{N}(t+1)
\end{array}\right)=A\left(\begin{array}{c}
x_{1}(t) \\
x_{M}(t) \\
x_{N}(t) \\
y_{1}(t) \\
y_{M}(t) \\
y_{N}(t)
\end{array}\right) \text {, with }
$$

$$
A=\left(\begin{array}{cc}
A_{1}\left(x(t)+y(t), \phi^{*}\right) & 0 \\
0 & A_{1}\left(x(t)+y(t), \phi^{\prime}\right)
\end{array}\right), \text { and } A_{1}(x, \phi)=\left(\begin{array}{ccc}
0 & f_{M}\left(x_{M}\right) & f_{N}\left(x_{N}\right) \\
\phi s_{M} & 0 & 0 \\
(1-\phi) s_{N} & 0 & 0
\end{array}\right)
$$

Let $(\tilde{x}, \tilde{y})$ be a positive fixed point of $(32)$. 
If we set

$$
\begin{aligned}
& z_{1}=s_{M}\left(\phi^{*} \tilde{x}_{1}+\phi^{\prime} \tilde{y}_{1}\right) \\
& z_{2}=s_{N}\left(\left(1-\phi^{*}\right) \tilde{x}_{1}+\left(1-\phi^{\prime}\right) \tilde{y}_{1}\right),
\end{aligned}
$$

then there must hold in particular, that:

$$
\left(\begin{array}{cc}
s_{M} \phi^{*} & s_{N}\left(1-\phi^{*}\right) \\
s_{M} \phi^{\prime} & s_{N}\left(1-\phi^{\prime}\right)
\end{array}\right)\left(\begin{array}{l}
f_{M}\left(z_{1}\right) \\
f_{N}\left(z_{2}\right)
\end{array}\right)=\left(\begin{array}{l}
1 \\
1
\end{array}\right)
$$

where we have used that $\tilde{x}_{1}$ and $\tilde{y}_{1}$ are both positive. Let $B=\left(\begin{array}{cc}s_{M} \phi^{*} & s_{N}\left(1-\phi^{*}\right) \\ s_{M} \phi^{\prime} & s_{N}\left(1-\phi^{\prime}\right)\end{array}\right)$, and notice that $\operatorname{det}(B)=s_{M} s_{N}\left(\phi^{*}-\phi^{\prime}\right) \neq 0$ since $\phi^{\prime} \neq \phi^{*}$. Hence, the system (35) can have at most one solution $\left(z_{1}, z_{2}\right)$ because both functions $f_{M}$ and $f_{N}$ are decreasing by hypothesis (H1), and are therefore 1-to-1 functions. Equations $(33)-(34)$ can be re-written as follows:

$$
\left(\begin{array}{cc}
s_{M} \phi^{*} & s_{M} \phi^{\prime} \\
s_{N}\left(1-\phi^{*}\right) & s_{N}\left(1-\phi^{\prime}\right)
\end{array}\right)\left(\begin{array}{l}
\tilde{x}_{1} \\
\tilde{y}_{1}
\end{array}\right)=\left(\begin{array}{l}
z_{1} \\
z_{2}
\end{array}\right)
$$

Similarly, (36) has at most one solution $\left(\tilde{x}_{1}, \tilde{y}_{1}\right)$. Since the other components of a fixed point are uniquely determined by $\tilde{x}_{1}$ and $\tilde{y}_{1}$ (namely, $\tilde{x}_{M}=s_{M} \phi^{*} \tilde{x}_{1}, \tilde{x}_{N}=s_{N}\left(1-\phi^{*}\right) \tilde{x}_{1}$ and $\left.\tilde{y}_{M}=s_{M} \phi^{\prime} \tilde{y}_{1}, \tilde{y}_{N}=s_{N}\left(1-\phi^{\prime}\right) \tilde{y}_{1}\right)$, we have just shown that the coupled system (32) can have at most one positive fixed point.

\subsection{Proof of Theorem 4.2.}

Proof. Step 1: We search for the fixed points $(x, \phi)$ of system $(15)-(16)$.

If we assume that $x_{1}=0$, then $x_{M}=x_{N}=0$ as well. But then the fixed point equation associated to equation (16) implies that $s_{M} f_{M}(0)-s_{N} f_{N}(0)=0$, contradicting (17). Hence, we assume that the fixed point $(x, \phi)$ is such that $x_{1}>0$. Then a similar argument implies that

$$
s_{M} f_{M}\left(x_{M}\right)=s_{N} f_{N}\left(x_{N}\right)
$$

must hold, and using $x_{1}>0$, that:

$$
1=\phi\left(s_{M} f_{M}\left(x_{M}\right)-s_{N} f_{N}\left(x_{N}\right)\right)+s_{N} f_{N}\left(x_{N}\right)
$$

The last two equations imply that

$$
s_{M} f_{M}\left(x_{M}\right)=1=s_{N} f_{N}\left(x_{N}\right),
$$

but since $f_{M}$ and $f_{N}$ are decreasing functions, the values of $x_{M}$ and $x_{R}$ are uniquely determined:

$$
x_{M}=\hat{x}_{M} \text { and } x_{N}=\tilde{x}_{N},
$$

see Theorem 2.1. Then it follows that

$$
\phi=\phi^{*},
$$

where $\phi^{*}$ was defined in formula (8). Finally,

$$
x_{1}=\hat{x}_{M} /\left(\phi^{*} s_{M}\right)\left(=\tilde{x}_{N} /\left(\left(1-\phi^{*}\right) s_{N}\right) .\right.
$$

We have shown that there is a unique fixed point for system $(15)-(16)$, which equals the vector $\left(x^{*}\left(\phi^{*}\right), \phi^{*}\right)$, where $x^{*}\left(\phi^{*}\right)$ is the unique positive fixed point of system (1) in case $\phi=\phi^{*}$.

Step 2: To prove local asymptotic stability of the fixed point $\left(x^{*}\left(\phi^{*}\right), \phi^{*}\right)$ provided that $\sigma^{2}>0$ is sufficiently small, we linearize the Darwinian system $(15)-(16)$ near the fixed point $\left(x^{*}\left(\phi^{*}\right), \phi^{*}\right)$ yielding the following Jacobian matrix:

$$
J_{D}\left(\sigma^{2}\right)=\left(\begin{array}{cc}
C\left(x^{*}\left(\phi^{*}\right), \phi^{*}\right) & * \\
0 & 1
\end{array}\right)+\sigma^{2}\left(\begin{array}{cccc}
0 & 0 & 0 & 0 \\
0 & 0 & 0 & 0 \\
0 & 0 & 0 & 0 \\
0 & \frac{s_{M} f_{M}^{\prime}\left(x_{M}^{*}\left(\phi^{*}\right)\right)}{2} & -\frac{s_{N} f_{N}^{\prime}\left(x_{N}^{*}\left(\phi^{*}\right)\right)}{2} & 0
\end{array}\right),
$$


where the $*$ represents a 3 -dimensional vector whose value is unimportant at present, and the $3 \times 3$ nonnegative matrix $C\left(x^{*}\left(\phi^{*}\right), \phi^{*}\right)$ is defined as:

$$
C\left(x^{*}\left(\phi^{*}\right), \phi^{*}\right)=A_{1}\left(x^{*}\left(\phi^{*}\right), \phi^{*}\right)+\left(\begin{array}{ccc}
0 & f_{M}^{\prime}\left(x_{M}^{*}\left(\phi^{*}\right)\right) x_{M}^{*}\left(\phi^{*}\right) & f_{N}^{\prime}\left(x_{N}^{*}\left(\phi^{*}\right)\right) x_{N}^{*}\left(\phi^{*}\right) \\
0 & 0 & 0 \\
0 & 0 & 0
\end{array}\right)
$$

By $(\mathbf{H 1})$, it follows that the nonzero entries in the second matrix on the right, are both negative. Moreover, since $A_{1}\left(x^{*}\left(\phi^{*}\right), \phi^{*}\right) x^{*}\left(\phi^{*}\right)=x^{*}\left(\phi^{*}\right)$, it follows that the dominant eigenvalue of the non-negative matrix $A_{1}\left(x^{*}\left(\phi^{*}\right), \phi^{*}\right)$ equals 1 , and hence the Perron-Frobenius Theorem implies that the dominant eigenvalue of the non-negative matrix $C\left(x^{*}\left(\phi^{*}\right), \phi^{*}\right)$ is less than 1 . Consequently, the dominant eigenvalue of $J_{D}(0)$ is 1 , and this eigenvalue is simple. By continuity of eigenvalues, the matrix $J_{D}\left(\sigma^{2}\right)$ will also have a real, simple and dominant eigenvalue $\lambda_{p}\left(\sigma^{2}\right)$ for all sufficiently small $\sigma^{2}$, such that $\lambda_{p}(0)=1$. We claim that $\lambda_{p}\left(\sigma^{2}\right)<1$, at least for all sufficiently small $\sigma^{2}$. To prove this, we now examine the roots of the characteristic polynomial $F\left(\lambda, \sigma^{2}\right):=\operatorname{det}\left(\lambda I-J_{D}\left(\sigma^{2}\right)\right)$ associated to the matrix $J_{D}\left(\sigma^{2}\right)$. To avoid heavy notation, we first recall the definitions of the functions $g_{M}$ and $g_{N}$ in $(\mathbf{H 2})$, and define positive constants $a, b, c, d, g$ and $h$, and negative constants $e$ and $f$, as follows:

$$
J_{D}\left(\sigma^{2}\right)=\left(\begin{array}{cccc}
0 & a & b & 0 \\
c & 0 & 0 & g \\
d & 0 & 0 & -h \\
0 & \sigma^{2} e & -\sigma^{2} f & 1
\end{array}\right):=\left(\begin{array}{cccc}
0 & g_{M}^{\prime}\left(x_{M}^{*}\left(\phi^{*}\right)\right) & g_{N}^{\prime}\left(x_{N}^{*}\left(\phi^{*}\right)\right) & 0 \\
\phi^{*} s_{M} & 0 & 0 & s_{M} x_{1}^{*}\left(\phi^{*}\right) \\
\left(1-\phi^{*}\right) s_{N} & 0 & 0 & -s_{N} x_{1}^{*}\left(\phi^{*}\right) \\
0 & \sigma^{2} \frac{s_{M} f_{M}^{\prime}\left(x_{M}^{*}\left(\phi^{*}\right)\right)}{2} & -\sigma^{2} \frac{s_{N} f_{N}^{\prime}\left(x_{N}^{*}\left(\phi^{*}\right)\right)}{2} & 1
\end{array}\right)
$$

A tedious calculation shows that the characteristic polynomial of the matrix $J_{D}\left(\sigma^{2}\right)$ is given by:

$$
F\left(\lambda, \sigma^{2}\right)=\lambda^{4}-\lambda^{3}-\left[\sigma^{2}(e g+f h)+(a c+b d)\right] \lambda^{2}+(a c+b d) \lambda+\sigma^{2}(a f+b e)(c h+d g)
$$

Note that $F(\lambda, 0)$ is positive for all $\lambda>1=\lambda_{p}(0)$ (since $\lambda_{p}(0)=1$ is the dominant root of $F(\lambda, 0)$, and $\left.\lim _{\lambda \rightarrow \infty} F(\lambda, 0)=+\infty\right)$. Moreover, $\partial F / \partial \lambda\left(\lambda_{p}(0), 0\right)$ must be positive because $\lambda_{p}(0)=1$ is a simple root of $F(\lambda, 0)$ (this can also be shown directly by calculating this partial derivative using the expression above: $\partial F / \partial \lambda\left(\lambda_{p}(0), 0\right)=1-(a c+b d)$, and this is positive because $a c+b d$ is the basic reproduction number associated to the matrix $C\left(x^{*}\left(\phi^{*}\right), \phi^{*}\right)$ which is less than 1 , as shown earlier). Therefore, the claim above (namely, that $\lambda_{p}\left(\sigma^{2}\right)<1$, for all sufficiently small $\sigma^{2}$ ) will be proved, provided we can show that for all sufficiently small $\sigma^{2}$, there holds that:

$$
F\left(1, \sigma^{2}\right)>1
$$

Evaluating $F\left(1, \sigma^{2}\right)$ yields:

$$
F\left(1, \sigma^{2}\right)=\sigma^{2}((a f+b e)(c h+d g)-(e g+f h)) .
$$

Therefore, a sufficient condition for (38) to hold, is that:

$$
f[a(c h+d g)-h]+e[b(c h+d g)-g]>0
$$

Recall that $e$ and $f$ are negative, which implies that the above inequality holds if:

$$
c h+d g<\frac{h}{a} \text { and } c h+d g<\frac{g}{b}
$$

or equivalently, by using the definitions of the various parameters in (37), if:

$$
s_{M} g_{M}^{\prime}\left(x_{M}^{*}\left(\phi^{*}\right)\right)<1 \text { and } s_{N} g_{N}^{\prime}\left(x_{N}^{*}\left(\phi^{*}\right)\right)<1 \text {. }
$$

Recalling the definition of the functions $g_{M}$ and $g_{N}$ in $(\mathbf{H 2})$, this holds if:

$$
s_{M}\left(f_{M}^{\prime}\left(x_{M}^{*}\left(\phi^{*}\right)\right) x_{M}^{*}\left(\phi^{*}\right)+f_{M}\left(x_{M}^{*}\left(\phi^{*}\right)\right)\right)<1 \text { and } s_{N}\left(f_{N}^{\prime}\left(x_{N}^{*}\left(\phi^{*}\right)\right) x_{N}^{*}\left(\phi^{*}\right)+f_{N}\left(x_{N}^{*}\left(\phi^{*}\right)\right)\right)<1
$$

However, we have shown in Step 1 that $s_{M} f_{M}\left(x_{M}^{*}\left(\phi^{*}\right)\right)=1=s_{N} f_{N}\left(x_{N}^{*}\left(\phi^{*}\right)\right)$, and thus the above inequality holds, provided that:

$$
s_{M} f_{M}^{\prime}\left(x_{M}^{*}\left(\phi^{*}\right)\right) x_{M}^{*}\left(\phi^{*}\right)<0 \text { and } s_{N} f_{N}^{\prime}\left(x_{N}^{*}\left(\phi^{*}\right)\right) x_{N}^{*}\left(\phi^{*}\right)<0 .
$$

By (H1), these last two inequalities are indeed satisfied. This concludes the proof of this Theorem. 


\section{REFERENCES}

[1] N. M. A. Allen, B. And U. Dieckmann, Adaptive dynamics with interaction structure, The American Naturalist, 181 (2013), pp. E139-E163.

[2] R. Beverton and S. Holt, On the Dynamics of Exploited Fish Populations, 1957.

[3] R. V. Bulkley, Fecundity of Steelhead Trout, Salmo gairdneri, from Alsea River, Oregon, Journal of the Fisheries Research Board of Canada, 24 (1967), pp. 917-926.

[4] H. Caswell, Matrix Population Models, Sinauer Associates, Sunderland, Mass, 2nd edition ed., Sept. 2000.

[5] B. B. Chapman, C. Brnmark, J.-. Nilsson, And L.-A. Hansson, The ecology and evolution of partial migration, Oikos, 120 (2011), pp. 1764-1775.

[6] F. Christiansen, On conditions for evolutionary stability for a continuously varying character, The American Naturalist, 138 (1991), pp. 37-50.

[7] J. Cushing, An introduction to structured population dynamics, SIAM, 1998.

[8] O. Diekmann, A beginner's guide to adaptive dynamics, Banach Center Publications, 63 (2004), pp. 47-86.

[9] J. J. Dodson, N. Aubin-Horth, V. Thriault, And D. J. Pez, The evolutionary ecology of alternative migratory tactics in salmonid fishes, Biological Reviews, 88 (2013), pp. 602-625.

[10] I. Eshel, Evolutionary and continuous stability, Journal of Theoretical Biology, 103 (1983), pp. 99-111.

[11] T. E. Essington, T. P. Quinn, And V. E. Ewert, Intra-and inter-specific competition and the reproductive success of sympatric Pacific salmon, Canadian Journal of Fisheries and Aquatic Sciences, 57 (2000), pp. 205-213.

[12] A. P. Foss-Grant, E. F. Zipkin, J. T. Thorson, O. P. Jensen, And W. F. Fagan, Hierarchical analysis of taxonomic variation in intraspecific competition across fish species, Ecology, (2016).

[13] C. K. Griswold, C. M. TAYlor, And D. R. NorRIs, The equilibrium population size of a partially migratory population and its response to environmental change, Oikos, 120 (2011), pp. 1847-1859.

[14] M. Hassell, Density-dependence in single-species populations, The Journal of Animal Ecology, 44 (1975), pp. $283-295$.

[15] M. W. Hirsch AND H. SMITH, Monotone maps: a review, Journal of Difference Equations and Applications, 11 (2005), pp. 379-398.

[16] A. Kaitala, V. Kaitala, and P. Lundberg, A Theory of Partial Migration, American Naturalist, 142 (1993), pp. 5981. WOS:A1993LW99900004.

[17] N. W. Kendall, J. R. McMillan, M. R. Sloat, T. W. Buehrens, T. P. Quinn, G. R. Pess, K. V. Kuzishchin, M. M. McClure, R. W. Zabel, And M. Bradford, Anadromy and residency in steelhead and rainbow trout ( Oncorhynchus mykiss ): a review of the processes and patterns, Canadian Journal of Fisheries and Aquatic Sciences, 72 (2015), pp. 319-342.

[18] H. KoKKO, Modelling for field biologists and other interesting people, Cambridge University Press, 2007.

[19] K. V. Kuzishchin, A. Y. Maltsev, M. A. Gruzdeva, K. A. Savvaitova, D. S. Pavlov, And D. A. Stanford, On joint spawning of anadromous and resident mykiss Parasalmo mykiss in rivers of Western Kamchatka, Journal of Ichthyology, 47, pp. 348-352.

[20] R. LANDE, Natural selection and random genetic drift in phenotypic evolution, Evolution, 30 (1976), pp. $314-334$.

[21] — A quantitative genetic theory of life history evolution, Ecology, 63 (1982), pp. 607-615.

[22] C.-K. Li And H. Schneider, Applications of Perron-Frobenius theory to population dynamics, Journal of Mathematical Biology, 44 (2002), pp. 450-462.

[23] P. Lundberg, The evolution of partial migration in birds, Trends in Ecology and Evolution, 3 (1988), pp. $172-175$.

[24] P. Lundberg, On the evolutionary stability of partial migration, Journal of Theoretical Biology, 321 (2013), pp. 36 - 39.

[25] M. G. Mitro And A. V. ZAle, Seasonal Survival, Movement, and Habitat Use of Age-0 Rainbow Trout in the Henrys Fork of the Snake River, Idaho, Transactions of the American Fisheries Society, 131 (2002), pp. 271-286.

[26] J. W. Moore, D. E. Schindler, And C. P. RufF, Habitat saturation drives thresholds in stream subsidies, Ecology, 89 (2008), pp. 306-312.

[27] D. S. Pavlov, N. N. Nemova, P. I. Kirillov, E. A. Kirillova, Z. A. Nefedova, And O. B. Vasileva, The lipid status and feeding habits of yearlings of mykiss Parasalmo mykiss, Journal of Ichthyology, 50 (2010), pp. 543-551.

[28] C. Perez, J. P. Granadeiro, M. P. Dias, H. Alonso, and P. Catry, When males are more inclined to stay at home: insights into the partial migration of a pelagic seabird provided by geolocators and isotopes, Behavioral Ecology, 25 (2013), pp. 313-319.

[29] F. PULIDO, Evolutionary genetics of partial migration-the threshold model of migration revis(it)ed, Oikos, 120 (2011), pp. $1776-1783$.

[30] T. P. Quinn, The behavior and ecology of Pacific salmon and trout, University of Washington Press, Bethesda, MD, 2005.

[31] T. P. Quinn, T. R. Seamons, L. A. Vllestad, And E. Duffy, Effects of growth and reproductive history on the egg sizefecundity trade-off in steelhead, Transactions of the American Fisheries Society, 140 (2011), pp. 45-51.

[32] W. Ricker, Stock and recruitment, Journal of the Fisheries Research Board of Canada, 11 (1954), pp. 559-623.

[33] R. Russell, Rainbow trout life history studies in lower Talarik Creek - Kvichak drainage, Tech. Rep. G-II-E, Alaska Department of Fish and Game, 1977.

[34] G. M. S.A.H. Geritz, E. KISDI AND J. MEtz, Evolutionary singular strategies and the adaptive growth and branching of the evolutionary tree, Evolutionary Ecology, 12 (1998), pp. 35-57. 
[35] W. H. Satterthwaite, M. P. Beakes, E. M. Collins, D. R. Swank, J. E. Merz, R. G. Titus, S. M. Sogard, and M. Mangel, Steelhead Life History on California's Central Coast: Insights from a State-Dependent Model, Transactions of the American Fisheries Society, 138 (2009), pp. 532-548.

[36] W. H. Satterthwaite, M. P. Beakes, E. M. Collins, D. R. Swank, J. E. Merz, R. G. Titus, S. M. Sogard, and M. MANGEL, State-dependent life history models in a changing (and regulated) environment: steelhead in the California Central Valley, Evolutionary Applications, 3 (2010), pp. 221-243.

[37] D. J. Schill, G. W. LaBar, E. R. Mamer, And K. A. Meyer, Sex ratio, fecundity, and models predicting length at sexual maturity of redband trout in Idaho desert streams, North American Journal of Fisheries Management, 30 (2010), pp. $1352-1363$.

[38] R. Schroeder And L. Smith, Life history of rainobw trout and effects of angling regulations in the Deschutes River, Oregon Information report (fish) 89-6, 1989.

[39] L. Shapovalov AND A. C. TAFT, The life histories of the steelhead rainbow trout (Salmo gairdneri gairdneri) and silver salmon (Oncorhynchus kisutch): with special reference to Waddell Creek, California, and recommendations regarding their management, California Department of Fish and Game, 1954.

[40] A. Shaw And S. Levin, To breed or not to breed: a model of partial migration, Oikos, 120 (2011), pp. 1871-1879.

[41] C. M. TAYLOR AND D. R. NorRIs, Predicting conditions for migration: effects of density dependence and habitat quality, Biology Letters, 3 (2007), pp. 280-284.

[42] E. P. van den Berghe And M. R. Gross, Natural Selection Resulting from Female Breeding Competition in a Pacific Salmon (Coho: Oncorhynchus kisutch), Evolution, 43 (1989), pp. 125-140.

[43] J. S. VANDERGRAFT, Spectral properties of matrices which have invariant cones, SIAM Journal of Applied Mathematics, 16 (1968), pp. 1208-1222.

[44] T. Vincent and J. Brown, Evolutionary Game Theory, Natural Selection, and Darwinian Dynamics, Cambridge, 2005.

[45] B. R. WARD, Declivity in steelhead (Oncorhynchus mykiss) recruitment at the Keogh River over the past decade, Canadian Journal of Fisheries and Aquatic Sciences, 57 (2000), pp. 298-306.

[46] B. R. Ward And P. A. Slaney, Egg-to-smolt survival and fry-to-smolt density dependence of Keough River steelhead trout, Canadian Special Publication of Fisheries and Aquatic Sciences, (1993), pp. 209-217.

Department of Mathematics, Oregon State University, Corvallis, OR 97330

Department of Mathematics, Oregon State University, Corvallis, OR 97330

Department of Integrative Biology, Oregon State University, Corvallis, OR 97330

Department of Integrative Biology, Oregon State University, Corvallis, OR 97330

Department of Mathematics, University of Arizona, Tuscon, AZ 85721 